Western University

Scholarship@Western

Applied Mathematics Publications

Applied Mathematics Department

9-1-2015

Sex allocation and the emergence of helping in cooperatively breeding species.

Josh D Dunn

Teodora Vujicic

Geoff Wild

Follow this and additional works at: https://ir.lib.uwo.ca/apmathspub

Part of the Applied Mathematics Commons, and the Ecology and Evolutionary Biology Commons

Citation of this paper:

Dunn, Josh D; Vujicic, Teodora; and Wild, Geoff, "Sex allocation and the emergence of helping in cooperatively breeding species." (2015). Applied Mathematics Publications. 5.

https://ir.lib.uwo.ca/apmathspub/5 


\title{
Sex allocation and the emergence of helping in cooperatively breeding species
}

\author{
Josh D. Dunn, Teodora Vujicic, and Geoff Wild* \\ Department of Applied Mathematics, The University of Western Ontario \\ London ON N6A 5B7, Canada \\ *Author for correspondence: gwild@uwo.ca
}

Dunn, J. D., Vujicic, T., and Wild, G. (2015). Sex allocation and the emergence of helping in cooperatively breeding species. Theoretical Population Biology, 104:1-9.

Publisher version of this article: http://dx.doi.org/10.1016/j.tpb.2015.06.006

This document is released with a Creative Commons Attribution Non-Commercial No Derivatives License

Keywords: alloparental care, helpers-at-the-nest, invasion analysis, kin selection, sex ratio, single-locus population genetics 


\begin{abstract}
In cooperative breeding systems individuals invest in the reproductive success of others. In this paper, we study the emergence of cooperative breeding systems in which reproductively active breeders receive investment from reproductively non-active helpers. Our goal is to understand how the division of an investment between male and female components of breeder fitness (i.e. the helper sex-allocation strategy) influences the emergence of cooperative breeding itself. Using mathematical models, we arrive at expressions for the inclusive-fitness advantage of helpful behaviour that generalize previous work. These expressions assume an ecologically stable environment, and that breeders make evolutionarily stable sex-allocation decisions. We find that, when breeders are extremely resource limited, the sex-allocation strategy used by a helper can be a key determinant in the success of helpful alleles. This finding, however, is restricted to cases in which helpers have access to intermediate levels of resources. Surprisingly, when helpers can make only a small investment in a recipient the division of the investment matters only very little to advantage of help. By contrast when resources are extremely abundant, we obtain the unsurprising result that the manner in which resources are allocated has little influence on the emergence of help. When breeders have access to intermediate levels of resources we find increasing relatedness can, in certain cases, inhibit the emergence of help. We also find that increasing the amount of resources available to a breeder can impede help as well. Both of these counter-intuitive results are mediated by evolutionary responses in breeder sex allocation.
\end{abstract}




\section{Introduction}

In a cooperative-breeding system, certain individuals promote the reproductive success of their neighbours, sometimes incurring large personal fitness costs as a result. In extreme cases, reproductive skew is high, and helpful individuals must postpone or even forgo their own reproduction to attend to offspring produced by a dominant breeder or breeding pair (Griffin and West, 2003).

Helping among cooperatively breeding individuals is known to influence the evolution of other social traits. In particular, sex allocation - the manner in which resources are divided between male and female components of reproductive fitness - is known to be affected by help (Emlen et al., 1986; Pen and Weissing, 2000a; Griffin et al., 2005; Wild, 2006). This influence is commonly observed as a sex-ratio bias in cooperatively breeding species, with investment favouring the more helpful sex.

Although the effect of helpful behaviour on sex allocation is quite well understood, the same cannot be said for the effect that sex allocation has on the emergence of helpful behaviour. What little we do know about the influence sex allocation has on helping in cooperative-breeding systems has come from studies by Taylor (1992) and Johnstone and Cant (2008). Using kin-selection models, both sets of authors outlined mathematical conditions for the advantage of helping among reproductively active neighbours. Sex allocation decisions did not factor into the conditions they developed, simply because any increase (resp. decrease) in investment made in a given sex was balanced by a decrease (resp. increase) in

the reproductive value of that sex. Importantly, Johnstone and Cant $(2008)$ did find that 
sex-specific patterns of dispersal could influence the emergence of helping. Consequently, changes to sex allocation could influence helping indirectly by changing evolutionarily stable dispersal rates (e.g. see Wild and Taylor, 2004).

At first glance, then, it might seem reasonable to expect sex allocation to play only an indirect role in the emergence of cooperative breeding. However, none of the work that could support such an expectation (i.e., Taylor, 1992, Johnstone and Cant, 2008) allowed the donor itself to influence the allocation of its investment. Given that donor and recipient can have different social-evolutionary perspectives in general (e.g. as in social insects: Trivers and Hare, 1976), it seems more reasonable to expect sex allocation could be "tuned" to maximize the donor's incentive to help. In other words, a direct role for sex allocation in the emergence of help ultimately appears plausible, despite the conclusions of previous studies.

In order to outline how sex allocation might directly influence the emergence of cooperative breeding systems, we generalized two recent models (Wild and Koykka, 2014) to include variable investment in male/female components of reproductive success and inbreeding. Our models assume a high-degree of reproductive skew, in the sense that they track the invasion of an allele that leads one individual to delay its own chance at reproduction in order to improve the reproductive success of another.

As expected, we can easily identify a direct role for sex allocation in the emergence of cooperative breeding. Specifically, we find that conditions for the advantage of help can be made more/less stringent by altering the way in which a donor divides its investment between the male and female components of recipient fitness. More importantly, we show why one should expect such dependence to occur: selection on breeder allocation implies that maximum advantage is achieved by a helper who allocates its resources like resource- 
unlimited breeders. Finally, we evaluate how competition among kin can alter this basic result, and the consequences of following what parent does for the emergence of help.

The sections that follow describe our models and results in three parts. The first part details the population dynamics that underlie our evolutionary argument. In the second part we determine the evolutionarily stable (ES) sex-allocation strategy used by a breeder in the absence of help. And in the third part we develop the conditions for the advantage of help offered to a breeder with ES sex allocation.

\section{Population Dynamics}

Although we extend Wild and Koykka (2014), the population dynamics that underlie our model follow theirs closely. We begin by considering a population whose individuals do not engage in any form of helpful behaviour. These individuals are diploid, sexual, and (for mathematical convenience) simultaneous hermaphrodites.

There are two different kinds of individuals in a genetically monomorphic population: breeders and floaters. Breeders are reproductively active individuals. Each breeder is associated with one breeding territory, and no two breeders share territories. By contrast, floaters are not reproductively active, though they are reproductively capable. Floaters are not associated with any breeding territory.

We census the population at discrete, evenly-spaced points in time. Let $F(t)$ and $B(t)$ denote the number of floaters and breeders, respectively, censused at the beginning of time step $t$. Following census, each breeder produces one viable oocyte with probability $p_{q}$. If viable, the oocyte is fertilized by some breeder in the population. With probability $\phi$ self- 
fertilization occurs, and with probability $1-\phi$ fertilization is accomplished by a uniform random breeder in the population. The resulting offspring becomes either a breeder or a floater in the next time step depending on the specific model scenario (No Territory Inheritance or Territory Inheritance, described below).

Each breeder survives from one time step to the next with probability $s_{b}$. When a breeder survives, it retains its breeding territory, and any offspring produced by that breeder disperses to become a floater. When a breeder dies, one of two things can occur. In our No Territory Inheritance (NTI) Model, we assume that offspring dispersal precedes all breeder mortality events. In this case offspring cannot fill a vacancy left by a dead parent; instead, offspring disperse to become floaters, and vacated territories simply vanish. In our Territory Inheritance (TI) Model, we assume that all breeder mortality events precede offspring dispersal. In this case, an offspring will fill a vacancy left by a dead parent rather than dispersing to become floaters. Territory inheritance is an important incentive for helping in cooperative-breeding systems (Stacey and Ligon, 1991). More importantly (as the reader will see) the TI model will allow us to consider the effects of competition among kin, whereas the NTI model will not.

Floaters survive from one season to the next with probability $s_{f}$. A fraction of the floaters that survive become breeders; the remaining fraction stay in the floater class. The probability that a surviving floater becomes a breeder is modelled as $1 /(1+a B(t))$ for some constant $a>0$, which is essentially Beverton-Holt density dependent competition (Britton, 2003). This is a decreasing function of the number of breeders $B(t)$, and so reflects an important kind of ecological constraint faced by cooperative breeders (Emlen, 1982a b).

The size of the population in the long-run is determined by a particular combination of 
parameters. This combination is called the basic reproduction number $\left(\mathcal{R}_{0}\right)$, and in this model it describes the expected number of newly established breeding territories produced on an established breeding territory when population densities are low. For the NTI Model we have $\mathcal{R}_{0}=p_{\rho} s_{f} /\left(1-s_{b}\right)$, and for the TI Model we have $\mathcal{R}_{0}=p_{\rho} s_{f} s_{b} /\left(\left(1-s_{b}\right)\left(1-p_{q}\right)\right)$ (Wild and Koykka, 2014). It can be shown that, when $\mathcal{R}_{0}<1$, the population tends to extinction over time, but when $\mathcal{R}_{0}>1$ the numbers of breeders and floaters in the population, respectively, tend to positive steady-state values (Wild and Koykka, 2014, see Appendix A). We disregard the marginal case $\mathcal{R}_{0}=1$, here, and assume that $\mathcal{R}_{0}>1$.

\section{Breeder's ES Sex Allocation}

In this section we use the population dynamics above to develop an inclusive-fitness argument for the ES sex-allocation strategy used by a breeder in the absence of help. Our results hold for both the NTI Model and the TI Model, and they can be verified using more formal invasion analyses (Courteau and Lessard, 2000, see Appendices C-F). We use the population dynamic models (seen in Appendix A) to determine whether sex allocation strategies are evolutionarily stable. Alongside this more formal approach, the inclusive-fitness argument is used to explain the invasion condition biologically.

We treat the sex-allocation strategy used by a breeder as a continuous trait. The trait determines the fraction of resources a breeder devotes to reproduction through male function, and is controlled at a single autosomal locus. There are two alleles at the locus in question, namely resident and mutant, with additive effects on the trait. Additivity allows us to think of a resident as having one sex-allocation strategy, $\alpha$, and of a mutant as having another 
sex-allocation strategy, $\beta$ (Taylor and Frank, 1996)

We suppose that each breeder has $k_{b}$ resources (e.g., measured in terms of calories) to allocate to either male or female reproductive function. Allocation to female function costs $c_{\varphi}$ (e.g., measured as calories per unit of function), and allocation to male function costs $c_{\sigma^{*}}$. It follows that a breeder using strategy $X=\alpha, \beta$ is able to "purchase" $k_{b}(1-X) / c_{q}$ units of female reproductive function, and $k_{b} X / c_{\sigma^{*}}$ units of male reproductive function.

Until now, the probability with which a breeder produced a viable oocyte has been treated as a constant, $p_{\text {}}$. In addition, all breeders had been assumed to compete on an equal basis for (non-self) fertilizations; a breeder's relative competitive ability is $p_{\sigma^{*}}$. Now, we treat $p_{\varphi}$ and $p_{\sigma^{*}}$ as a function of $k_{b}(1-X) / c_{q}$ and $k_{b} X / c_{\sigma^{*}}$, respectively. We consider two different paradigms for the functional forms of these reproductive functions, namely diminishing returns and logistic. In the diminishing returns paradigm, each unit of investment in reproductive function does less than the previous unit. Mathematically,

$$
p_{\varphi} \equiv p_{\varphi}(X)=1-\exp \left\{-\frac{k_{b}}{c_{\varphi}}(1-X)\right\} \quad \text { and } \quad p_{\sigma^{\prime}} \equiv p_{\sigma^{\prime}}(X)=1-\exp \left\{-\frac{k_{b}}{c_{\sigma^{\prime}}} X\right\}
$$

In the logistic paradigm, $p_{\phi}$ and $p_{\sigma^{\prime}}$ have regions of increasing and then diminishing rates of return on investment. Mathematically,

$$
p_{\varphi}(X)=\left[1+\left(\frac{1}{\varepsilon_{\varphi}}-1\right) e^{-k_{b}(1-X) / c_{\varphi}}\right]^{-1} \quad \text { and } \quad p_{\sigma^{\prime}}(X)=\left[1+\left(\frac{1}{\varepsilon_{\sigma^{\circ}}}-1\right) e^{-k_{b} X / c_{c^{\circ}}}\right]^{-1}
$$

where $p_{\varphi}(1)=\varepsilon_{q}$ and $p_{\sigma^{\circ}}(0)=\varepsilon_{\sigma^{*}}$ (i.e., no resources are allocated towards either female or male reproductive function, respectively). A comparison of the diminishing returns and logistic paradigms can be seen in Figure 1 .

Following Shaw and Mohler (1953), if $W(X, \alpha)$ is the fitness of an $X$-strategist in a 
population otherwise fixed for the resident strategy, then

$$
W(X, \alpha)=r_{b, q} \frac{p_{\wp}(X)}{p_{\wp}(\alpha)}+r_{b, \sigma^{*}}(1-\phi) \frac{p_{\sigma^{*}}(X)}{p_{\text {ơ }^{*}}(\alpha)}, \quad(X=\alpha, \beta)
$$

where $r_{b, q}=(1+\phi) / 2$ and $r_{b, \sigma^{*}}=1 / 2$ express the relatedness between a breeder and its reproductive output through female and male function, respectively (Appendix B). The term $(1-\phi)$ appears in equation 1 , because a breeder can only achieve reproductive success through male function when another breeder reproduces via outcrossing (probability $1-\phi)$.

The mutant strategy will invade whenever $W(\beta, \alpha)>W(\alpha, \alpha)=1$. However, it is enough to focus on $W(\beta, \alpha)$ when determining the ES breeder trait, $\alpha^{*}$. If $\alpha^{*}$ (between zero and one) is an ES trait, then in the diminishing returns paradigm

$$
\left.\frac{\partial W(\beta, \alpha)}{\partial \beta}\right|_{\beta=\alpha=\alpha^{*}}=-r_{b, q}\left(\frac{k_{b}}{c_{\varphi}}\right) \frac{1-p_{\varphi}\left(\alpha^{*}\right)}{p_{\varphi}\left(\alpha^{*}\right)}+r_{b, \sigma^{*}}(1-\phi)\left(\frac{k_{b}}{c_{\sigma^{*}}}\right) \frac{1-p_{\sigma^{*}}\left(\alpha^{*}\right)}{p_{\sigma^{*}}(\alpha)}=0,
$$

and in the logistic paradigm

$$
\left.\frac{\partial W(\beta, \alpha)}{\partial \beta}\right|_{\beta=\alpha=\alpha^{*}}=-r_{b, q}\left(\frac{k_{b}}{c_{q}}\right)\left(1-p_{\varphi}\left(\alpha^{*}\right)\right)+r_{b, \sigma^{*}}(1-\phi)\left(\frac{k_{b}}{c_{\sigma^{*}}}\right)\left(1-p_{\sigma^{*}}\left(\alpha^{*}\right)\right)=0
$$

(Taylor, 1996; Hamilton, 1964). In general, we must solve equations (2) and (3) numerically. We have also verified that in the diminishing returns paradigm solutions to (2) are evolutionarily stable - using the population dynamic models - in the sense that they cannot be invaded by rare mutants (Maynard Smith and Price, 1973), and in the sense that they are evolutionary attractors (see Appendices D and E) Christiansen, 1991; Courteau and Lessard, 2000).

In the logistic paradigm the strategy $\alpha^{*}$ that satisfies equation (3) is only ES when

$$
\frac{1}{c_{\varphi}}\left(p_{\uparrow}\left(\alpha^{*}\right)-\frac{1}{2}\right)+\frac{1}{c_{\sigma^{*}}}\left(p_{\sigma^{*}}\left(\alpha^{*}\right)-\frac{1}{2}\right)>0
$$


We are guaranteed that $\alpha^{*}$ is ES when both $p_{\text {o }}$ and $p_{\sigma^{*}}$ are in the region of diminishing returns - above the dashed line in Figure 1. When both $p_{q}$ and $p_{\sigma^{*}}$ are in the region of increasing rates of return on investment, $\alpha^{*}$ is not evolutionarily stable, leading to an evolutionary branching point-below the dashed line in Figure 1. From here on out we will Fig 1 assume that $p_{\varphi}$ and $p_{\sigma^{*}}$ follow the diminishing returns paradigm.

In general we must resort to numerical solution of equation (2), but we can find solutions for particular cases. For example, when the cost of investing in male and female reproductive function is the same $\left(c_{q}=c_{\sigma^{*}}=c\right)$, we find

$$
\alpha^{*}=\frac{c}{k_{b}} \log \left\{\frac{\phi+\sqrt{\phi^{2}+\left(1-\phi^{2}\right) e^{k_{b} / c}}}{1+\phi}\right\} \text {. }
$$

When breeders are extremely resource limited $\left(k_{b} \rightarrow 0^{+}\right)$, we find

$$
\alpha^{*}=\frac{1-\phi}{2}, \quad \text { for all } c_{q} \text { and } c_{\sigma^{*}}
$$

which is Hamilton's (1967) well known local mate competition result. Finally, when resources are in extreme abundance $\left(k_{b} \rightarrow \infty\right)$ we find

$$
\alpha^{*}=\frac{c_{\sigma^{*}}}{c_{\sigma^{*}}+c_{\varphi}}
$$

which leads to $p_{\varphi}\left(\alpha^{*}\right)=p_{\sigma^{*}}\left(\alpha^{*}\right)=1-\exp \left\{-k_{b} /\left(c_{\sigma^{*}}+c_{\varphi}\right)\right\}$, essentially Fisher's (1930) result for the advantage of equal production of the sexes. These results are proven in Appendices D.4 (equation 5) and D.5 (equation 6) .

For intermediate values of $k_{b}$, numerical solution of (2) shows that equations (5) and (6) provide bounds on the ES sex-allocation strategy, $\alpha^{*}$ (Figure 2). Like the expression in Fig 2 equation (4), numerically determined values of $\alpha^{*}$ decrease with increasing self-fertilization rates, $\phi$. This relationship between $\alpha^{*}$ and $\phi$ makes good sense: an increased self-fertilization 
rate implies that opportunities for breeders to compete for, and subsequently achieve, reproductive success through male function become more limited, and so investment in male function becomes more wasteful. Numerically determined values of $\alpha^{*}$ also vary with costs $c_{\varphi}$ and $c_{\sigma^{*}}$. Specifically, ES investment in one sex increases as the relative cost of that sex decreases. Again, we see that the ES strategy shies away from wasteful investment.

\section{Emergence of Helpful Behaviour}

\section{Change in Inclusive-Fitness Due to Help}

Having established our understanding of a breeder's ES sex-allocation strategy, we are ready to investigate the emergence of helping. We consider a population that has achieved a steady state, and has settled on the ES sex allocation strategy for breeders, $\alpha^{*}$. Unlike the previous section, we will have to analyse the NTI Model and the TI Model separately. The results of the analyses are verified using population-genetic models in Appendix F.

We assume that helping is controlled by an autosomal locus that also controls natal dispersal. This new helping/dispersal locus is independent of the locus determining sex allocation, and has two alleles (again labelled mutant and resident). The resident allele results in no change to an offspring's dispersal behaviour. By contrast, the mutant allele causes an offspring to delay dispersal for one time step with some small probability. During the delay, a philopatric mutant offspring will help its associated breeder (i.e., its parent), but that same offspring's survival is not guaranteed (it survives the time step with probability $\left.s_{h}\right)$. 
A helper aids a breeder by increasing the breeder's reproductive success. We assume that a helper has $k_{h}$ resources that can be allocated to a breeder's male or female reproductive function. If $\gamma$ is the fraction of a helper's resources allocated to a breeder's male reproductive function, then with help a breeder's ability to compete for fertilizations changes from $p_{\sigma^{*}}\left(\alpha^{*}\right)$ to $q_{\sigma^{\circ}}\left(\alpha^{*}, \gamma\right)=1-\exp \left\{-\left(k_{b} \alpha^{*}+k_{h} \gamma\right) / c_{\sigma^{*}}\right\}$. Similarly, with help the probability with which a breeder produces a viable oocyte changes from $p_{q}\left(\alpha^{*}\right)$ to $q_{q}\left(\alpha^{*}, \gamma\right)=1-\exp \left\{-\left(k_{b}\left(1-\alpha^{*}\right)+\right.\right.$ $\left.\left.k_{h}(1-\gamma)\right) / c_{q}\right\}$.

To calculate the inclusive-fitness change (Taylor, 1992) of a mutant individual, call it $\Delta w$, we fix attention on a newborn individual who decides to remain on its natal territory and help its parent for one season only. The calculation, itself, breaks the focal newborn's inclusive fitness into into direct and indirect components (Brown, 1987). Direct components describe changes to an individual's survival, as well as changes to its production of descendant kin. Indirect components describe changes to an individual's production of non-descendant kin. We will begin with indirect benefits, then move on to direct costs and benefits.

For the NTI model we find that, by delaying dispersal to help, the mutant (a) receives an indirect inclusive-fitness benefit due to increased breeder reproductive success, (b) receives a direct benefit when it inherits its parent's territory (the name "No Territory Inheritance" refers to the fact that the dispersive resident phenotype is unable to inherit a parent's territory), and (c) pays a direct cost in the event that it dies before attempting to breed. Mathematically, 
NTI Model:

$$
\begin{gathered}
\Delta w=\underbrace{p_{\varphi}\left(\alpha^{*}\right)\left(r_{h, \uparrow} \frac{q_{\varphi}\left(\alpha^{*}, \gamma\right)-p_{\varphi}\left(\alpha^{*}\right)}{p_{\varphi}\left(\alpha^{*}\right)}+r_{h, \sigma^{*}}(1-\phi) \frac{q_{\sigma^{*}}\left(\alpha^{*}, \gamma\right)-p_{\sigma^{*}}\left(\alpha^{*}\right)}{p_{\text {o }^{*}}\left(\alpha^{*}\right)}\right)}_{\text {(a) indirect benefit }} \\
+\underbrace{s_{h}\left(1-s_{b}\right)\left(\frac{p_{\varphi}\left(\alpha^{*}\right)}{1-s_{b}}-1\right)}_{\text {(b) direct benfit }}-\underbrace{\left(1-s_{h}\right)}_{\text {(c) direct cost }}
\end{gathered}
$$

(b) direct benefit

where $r_{h, q}=(1+\phi)^{2} / 4$ expresses the relatedness between a helper and its parent's reproductive output via female function, and where $r_{h, \sigma^{*}}=(1+\phi) / 4$ expresses the relatedness between a helper and its parent's reproductive output via male function. When $\Delta w$ is positive (resp. negative) helping confers a net advantage (resp. disadvantage), and the mutant invades (resp. is eliminated). Equation (7) generalizes one presented in Pen and Weissing (2000b) (their equation 25), and in Wild and Koykka (2014) (their equation 3.1).

For the TI model, a mutant that delays dispersal in order to help its associated breeder again (a) receives an indirect inclusive-fitness benefit due to increased breeder reproductive success, (b) receives a direct benefit when it inherits its parent's territory, and (c) pays a direct cost in the event that it dies before attempting to breed. However, in the TI model there is a fourth effect, namely (d) an indirect effect due to kin competition. This fourth effect is felt when a helper displaces the offspring that would have inherited the parent's territory had the helper dispersed (recall that the "Territory Inheritance" model ensured that offspring could inherit a territory rather than disperse). Mathematically, 
TI Model:

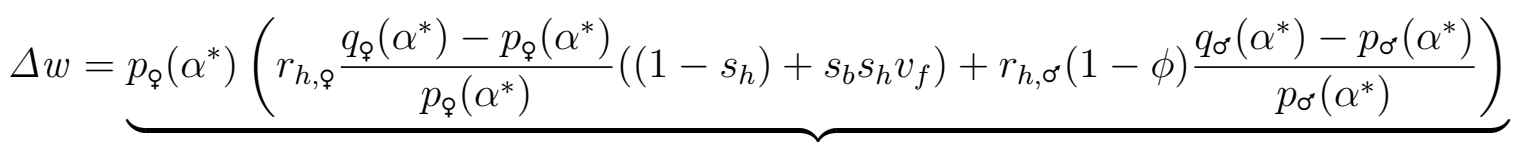

$$
\begin{aligned}
& \text { (a) indirect benefit } \\
& +\underbrace{s_{h}\left(1-s_{b}\right)\left(v_{i}-v_{f}\right)}_{\text {(b) direct benefit }}-\underbrace{\left(1-s_{h}\right) v_{f}}_{\text {(c) direct cost }}-\underbrace{s_{h}\left(1-s_{b}\right) r_{h, q}\left(p_{q}\left(\alpha^{*}\right) v_{i}-q_{q}\left(\alpha^{*}\right) v_{f}\right)}_{\text {(d) indirect cost/benefit }},
\end{aligned}
$$

where $v_{f}=\left(1-p_{\varphi}\left(\alpha^{*}\right)\right) / s_{b}$ is the reproductive value (sensu Fisher, 1930) of a resident floater, and $v_{i}=p_{\phi}\left(\alpha^{*}\right) /\left(1-s_{b}\right)$ is the reproductive value of a resident that inherits its parent's breeding territory. Equation (8) generalizes an expression presented by Wild and Koykka (2014) (their equation 3.2).

It is instructive to ask, what sex-allocation strategy exhibited by a helper would maximize the inclusive-fitness change due to helping? Applying elementary calculus to equations (7) and (8), respectively (Appendix G), we find that helping is promoted to the greatest extent when $\gamma$ is equal to

$$
\gamma_{\max }=\min \left\{\frac{c_{\sigma^{*}}}{c_{\sigma^{\prime}}+c_{q}}+\left(\frac{k_{h}}{c_{\sigma^{\prime \prime}}}+\frac{k_{h}}{c_{q}}\right)^{-1} \log \left(\frac{v_{\sigma^{*}}}{v_{q}}\right), 1\right\}
$$

where $v_{\sigma^{*}}=1$ is the reproductive value of success achieved through the breeder's male function, and $v_{\varphi}=1$ (NTI Model) or $v_{\varphi}=\left(1-s_{h}\right)+s_{h} v_{f}$ (TI Model) is the reproductive value of success achieved through the breeder's female function. The minimum appears in the expression for $\gamma_{\max }$ because it does not make biological sense for helper sex allocation to exceed one. From equation (9), we see that the advantage of helping is greatest when helpers in the NTI Model behave as resource-unlimited breeders (compare to equation 6), and when helpers in the TI Model behave as resource-unlimited breeders who reduce investment in 
female function because of kin competition (compare to equation 6, and notice that the second term in 9 is positive). To be clear, we do not claim that $\gamma_{\max }$ is the phenotype that helpers necessarily exhibit. Rather, we include $\gamma_{\max }$ here (i) to highlight the fact that the allocation strategy that best promotes the emergence of helping is, in general, not $\alpha^{*}$, and (ii) to serve as a reference point later, thus eliminating the need to consider $\gamma$ in numerical exploration of the model.

\section{Remarks on Resource Availability}

Helper sex allocation, $\gamma$, plays only a minor role in the emergence of helping whenever resources are in great abundance (either $k_{b}$ or $k_{h}$ large). In those cases, total investment in reproductive success is large enough that marginal returns on these investments are substantially diminished, and so it makes intuitive sense that the pattern of investment matters little to a helper's inclusive fitness. In fact, when resources are abundant, the emergence of helping is determined primarily by direct benefits (terms b-c in equations 7 and 8) and kin competition (term d in equation 8).

When only breeders are resource limited (or the cost of raising offspring independently is high), the expressions for the change in inclusive fitness due to help simplify. In this scenario, both equation (7) and equation (8) become,

NTI Model and TI Model $\left(k_{b} \rightarrow 0^{+}\right)$:

$$
\Delta w=\left(\frac{1+\phi}{2}\right)^{2}\left[\left(1-e^{-k_{h}(1-\gamma) / c_{\varphi}}\right)+\frac{c_{\sigma^{\prime}}}{c_{\phi}}\left(1-e^{-k_{h} \gamma / c_{\sigma^{\circ}}}\right)\right]-\left(1-s_{h}\right)
$$

where we have used $\mathcal{R}_{0}>1$ implies that $s_{b}$ must tend to 1 as $k_{b}$ becomes small (i.e. as $p_{q}\left(\alpha^{*}\right)$ becomes small). Notice that only the indirect benefit of helping and the direct cost 
of helping now feature in $\Delta w$. When resources are scarce, population dynamics imply that the positive and negative consequences of territory inheritance are negligible.

Turning our attention back to the indirect benefit and direct cost found in equation 10, we can make two simple observations about the emergence of help in the low breeder-resource limit. First, the emergence of helping is inhibited as the mortality of helpers (cost), $1-s_{h}$, is increased. Second, the emergence of helping is promoted by an increase in either the rate of self-fertilization (essentially, relatedness), $\phi$, or the amount of resources invested by a helper (essentially, the extent of the benefit), $k_{h}$.

In addition to the two basic observations above, Figure 3 shows that the emergence of $\mathrm{Fig} 3$ helping, as predicted by 10 , is promoted as the cost of investing in female function, $c_{\varphi}$, decreases. The effect of $c_{\varphi}$ makes sense in light of the fact that fitness gains through male function are limited by oocyte availability: in order to achieve a successful fertilization one needs something to fertilize. Figure 3 also shows that the effect of changing helper sexallocation, $\gamma$, is predicted to be modified by both the amount of resources available to a helper, and the sex-specific costs of investing in breeder reproductive success. In particular, we see that larger $k_{h}$ increases the range of $\gamma$ values over which helping is advantageous for a given cost (e.g., blue annotation in Figure 35). This agrees with our earlier finding that helper sex-allocation strategy plays almost no role in the emergence of help when resources are extremely abundant. At the other extreme, we see that sex allocation has a limited role to play in the emergence of help when $k_{h}$ is small. This is evidenced by the flattening of curves in Figure 3 as $k_{h}$ is decreased. Overall, the role of $\gamma$ is most prominent at intermediate levels of $k_{h}$. When helper sex allocation has a role to play, we see that increased allocation to male function promotes (resp. inhibits) the emergence of help when $\gamma$ is less than (resp. 
greater than) $c_{\sigma^{\prime}} /\left(c_{\sigma^{\circ}}+c_{q}\right)$ (Figure 3). This result agrees with the expression for $\gamma_{\max }$ in equation (9), for both the NTI and TI Models (for the latter take the limit as $k_{b}$ tends to zero).

As a supplement to Figure 3 we have included Figure 4 that shows the direct cost (helper mortality) as a function of the ES sex allocation and assumes that a helper is using the allocation strategy $\gamma_{\max }$. In accordance with Figure 3, Figure 4 also demonstrates that as $\operatorname{Fig} 4$ the cost of female units of reproductive function decrease (resp. increase) helping is promoted (resp. inhibited). In addition, Figure 4 shows that helping is promoted as more resources are allocated towards female units of reproductive function, and in this scenario $\left(k_{b} \rightarrow 0^{+}\right)$ since $\alpha^{*}=(1-\phi) / 2$, a smaller $\alpha^{*}$ corresponds to increased relatedness.

\section{Intermediate Resource Availability}

In this section we relax the assumption that breeders are extremely resource limited, and consider intermediate values of $k_{b}$.

In this case, we find that increasing self-fertilization rate $(\phi)$ does not always increase the scope for the emergence of helping. Specifically, in the NTI Model larger $\phi$ can inhibit the emergence of help (Figure 5a). This result stems from the fact that larger $\phi$ promotes breeder investment in female function. In turn, increased breeder investment decreases the marginal returns to helpers through breeder female function. Although marginal returns on helper investment in male function are also increased, larger $\phi$ (i.e. more selfing) means that gains through male function are less frequently realized. In the TI Model, stronger inhibitory effects of increased $\phi$ are due to the negative effects of competition among relatives (Figure 
6 .).

Fig 6

We also find increasing $k_{b}$, itself, has mixed consequences for helping when breeders have greater access to resources. In the NTI model, increasing $k_{b}$ (when $c_{q}$ is sufficiently small) reduces the marginal benefits awarded to helpers as described immediately above (Figure 5). A similar effect occurs in the TI model, but increasing $k_{b}$ has the added effect, there, of decreasing the discrepancy between offspring survival with an without help-effectively limiting the costs of competition among relatives (Figure 6). It should be noted that the NTI result, here, is different from one reported by Wild and Koykka (2014). In Wild and Koykka (2014) increasing the probability of offspring production (e.g. through larger $k_{b}$ ) uniformly improved the advantage of helping. Investment in that paper, however, was not divided between males and females. Here, when increasing $k_{b}$ inhibits helping, it also increases investment in male function (Figure $2 a$ ). Consequently, increasing $k_{b}$ can diminish the direct benefit of helping via an evolutionarily labile breeder sex-allocation strategy.

Finally, we find that increasing helper resources $\left(k_{h}\right)$ increases the scope for the emergence of helping all around (figure not shown). This result mirrors the result obtained in the small$k_{b}$ limit.

\section{Discussion}

\section{Sex Allocation and the Advantage of Helping}

Our goal with this paper was to investigate the ways in which sex allocation influences the emergence of helping in cooperative breeding systems. Using an inclusive-fitness based 
model, we demonstrated that sex allocation can promote the emergence of helping in certain situations. Specifically, we found that resource availability provides an organizing principle that separates situations in which sex allocation plays a significant role in the emergence of help from those in which it does not. When resources are abundant (equivalently, overall costs of investment low) our model predicts that helper sex allocation strategy has little effect on the emergence of helping - it is direct-fitness effects that matter in these cases. By contrast, when resources are scarce (equivalently, overall costs of investment high) we find that helper sex allocation strategy can be a determining factor in the emergence of help. Although the result for the former case could likely have been developed with verbal reasoning alone, exposition of the latter case required a mathematical model to ensure appropriate limits were taken correctly.

Previous theoretical work has indicated that the sex allocation strategy adopted by a helpful individual will play a limited role in determining the advantage of help itself (Taylor, 1992; Johnstone and Cant, 2008). That work, however, was based on the standard assumption that breeders produce large numbers of offspring; in other words, breeders have large amounts of resource at their disposal. Given that our model predicts a role for sex allocation only in cases where resources are limited, we suggest that resource scarcity is responsible for the difference between our predictions and those made elsewhere.

Our finding that helper sex allocation plays a significant role in the emergence of help in resource-limited species makes one important prediction about helper behaviour in the field. Emlen (1982a) argued that two very different kinds of ecological constraints contribute to the emergence of cooperative breeding systems. He drew a distinction between constraints that reduce the probability of establishing a breeding territory (e.g. habitat saturation, availabil- 
ity of mates), and those that limit the production of offspring once established (e.g. large cost of raising offspring, equivalently insufficient resources to raise offspring). Emlen also emphasized that constraints like habitat saturation should predominate in stable environments, whereas those like high costs reproduction should predominate in fluctuating environments. Combining Emlen's arguments with those made here, we predict that helper sex allocation will have the greatest effect on helping in fluctuating environments where offspring production, not habitat saturation, is the predominant ecological constraint. Granted, our model includes habitat saturation, but the probability with which a floater becomes established in our model is always $1 / \mathcal{R}_{0}$ which need not be small when resources are scarce (i.e., in the $\left.\operatorname{limit} k_{b} \rightarrow 0^{+}\right)$.

\section{Helper's Perspective Versus Breeder's Perspective and Some Spec-}

\section{ulation}

In the course of analysing our model, we identified a helper sex-allocation strategy, $\gamma_{\max }$ in equation (9), that maximizes the advantage of help. We do not claim that this strategy will necessarily be adopted by helpers. In fact, it seems unlikely to us that a de novo mutation would simultaneously produce a helper and a maximally advantageous sex-allocation strategy. Still, the fact that $\gamma_{\max }$ differs from the ES breeder allocation strategy $\alpha^{*}$ may have implications for what kind genetic evidence for helping is sought.

Before launching into a possible implication of the difference between $\gamma_{\max }$ and $\alpha^{*}$, we should address one source of potential confusion. It may seem surprising that the strategy that maximizes helper's inclusive-fitness benefit is not the same as the ES breeder strategy 
given that relatedness leads both helpers and breeders to weigh success through male function and success through female function equally. After all, asymmetries in relatedness coefficients are often responsible for differing perspectives and conflict over sex allocation (e.g. Pen and Taylor, 2005: Wild and Taylor, 2005; Pen, 2006: Wild and West, 2009). Nevertheless, surprise is not warranted because $\gamma_{\max }$ is not an equilibrium result. Selection acts on breeder sex allocation in the absence of help, ultimately leading the population to express a strategy $\left(\alpha^{*}\right)$ that balances fitness returns through male function with those made through female function. When helpful behaviour initially arises, then, the most effective helpers will not have the same regard for sex-specific returns on fitness as that shown by the breeders they attend. Instead, the most effective helpers will act as resource-unlimited breeders (or resource unlimited breeders who express concern for kin competition, as in the TI Model), because selection has effectively taken care of concerns associated with resource scarcity by acting on breeders.

Given that we do not necessarily expect helpers to express $\gamma_{\max }$, then what significance does it hold? One might argue that cases in which cooperative breeding successfully emerged, are also likely to have been cases in which helpers had the ability to allocate resources in a manner that was different from breeders. Rather than helper behaviour co-opting an existing locus controlling breeder behaviour, successful invasions may have been supported by gene duplication events that allowed helpers to express behaviours closer to $\gamma_{\max }$. Even though present-day breeders and helpers may have very similar equilibrium behaviours, evidence for the "ghost of successful invasions past" might still be sought in their genomes. Of course, this is highly speculative, but interest in elucidating the genetic basis of helpful behaviour is building - buoyed by the fact that genetics plays a key part in the theoretical foundations of 
sociobiology (Thompson et al., 2013). Identifying vestigial gene duplication events, if they have occurred, could add strength to the prevailing gene-centred view of social evolution, and maybe even quiet related debates (e.g. Laland et al., 2014).

\section{Limitations and Future Work}

The evolutionary dynamics of sex allocation were changed by the functional forms associated with the reproductive functions - the diminishing returns and logistic paradigms. In this paper, we disregard the scenario that led to an evolutionary branching point or possibly split sex allocation - the logistic paradigm - and instead focus on ES sex allocation strategies given by assuming diminishing returns. In a population with split sex allocation some individuals would favour investment in male reproductive function and others would favour investment in female reproductive function compared to the average allocation strategy (Grafen, 1986). Exploring exactly how split sex allocation influences the emergence of cooperative breeding would be an interesting avenue for future research.

Assumptions like uniform territory quality, lack of inbreeding depression, and high reproductive skew could be relaxed and would certainly influence model predictions. We leave the investigation of the effects due to further elaboration of our model for future work. One important assumption - one that deserves discussion - is that of weak selection. We needed to invoke weak selection in order to develop the inclusive-fitness interpretation of the invasion condition (Taylor, 1989). Here, weak selection meant that the tendency to help (equivalently, the tendency to remain on one's natal site) was small. This is in contrast to the way in which some authors understand weak selection: as implying that the behaviour in question has a 
small impact on fitness when expressed (Wild and Traulsen, 2007). Although it has the advantage of allowing helping to effect appreciable benefits when expressed, our version of weak selection ignore synergistic effects due to helpers aiding helpful breeders (Grafen, 1985). Thus, our version of weak selection necessarily ignores benefits due to delayed reciprocity (Wiley and Rabenold, 1984), and any associated benefits that might be realized by adjusting sex allocation. Such benefits could be realized, for example, by a helper who invests more in a breeder's female function in an effort to secure a helper for itself in the event it inherits the territory. Although previous theoretical work has investigated the effect of delayed reciprocity in cooperative breeders (Kokko et al., 2001), it has not done so using an explicit population dynamic model as we have done here. Moreover, to our knowledge, no previous theoretical work has combined the possibility of delayed reciprocity and variable sex allocation. Future work should address this gap.

We have presented the resource availability, above, as an environmental variable that can help us organize our understanding about the effect helper sex allocation has on the emergence of helping. As resource availability changes, we have also seen that the relative importance of indirect and direct benefits of help change. When resources are abundant, our models predict that the advantage of helping will rely primarily on direct benefits like territory inheritance. However, when resources are scarce, our models predict that the advantage stems primarily from the indirect benefits associated with raising non-descendant kin. These two extremes - direct benefits on one hand, and indirect benefits on the other - frame much of the discussion among biologists about cooperative breeding (Stacey and Ligon, 1991; Pen and Weissing, 2000b; Clutton-Brock, 2002; Griffin and West, 2003). Future work could explore the extent to which resource availability could act as a broader organizing 
principle for cooperative breeders, one that provides an environmental mechanism capable of mediating a transition between importance of direct benefits and indirect benefits (and vice versa).

\section{Acknowledgements}

This work was supported by the Natural Sciences and Engineering Research Council of Canada. The authors thank Cody Koykka for helpful discussion.

\section{References}

Britton, N. F. (2003). Essential mathematical biology. Springer, London.

Brown, J. L. (1987). Helping and Communal Breeding in Birds. Monographs in Behavior and Ecology. Princeton University Press, Princeton, NJ.

Christiansen, F. B. (1991). On conditions for evolutionary stability for a continuously varying character. Am. Nat., 128:37-50.

Clutton-Brock, T. (2002). Breeding together: kin selection and mutualism in cooperative vertebrates. Science, 296:69-72.

Courteau, J. and Lessard, S. (2000). Optimal sex ratios in structured populations. J. Theor. Biol., 207:159-175.

Emlen, S. T. (1982a). The evolution of helping. I. an ecological constraints model. Am. Nat., 119:29-39. 
Emlen, S. T. (1982b). The evolution of helping. II. the role of behavioral conflict. Am. Nat., 119:40-53.

Emlen, S. T., Emlen, J. M., and Levin, S. A. (1986). Sex ratios in species with helpers-atthe-nest. Am. Nat., 127:1-8.

Fisher, R. A. (1930). The genetical theory of natural selection. Clarendon, Oxford, UK.

Grafen, A. (1985). Hamilton's rule OK. Nature, 318:310-311.

Grafen, A. (1986). Split sex ratios and the evolutionary origins of eusociality. J. Theor. Biol., 122(1):95-121.

Griffin, A. S., Sheldon, B. C., and West, S. A. (2005). Cooperative breeders adjust offspring sex ratios to produce helpful helpers. Am. Nat., 166:628-632.

Griffin, A. S. and West, S. A. (2003). Kin discrimination and the benefit of helping in cooperatively breeding vertebrates. Science, 302:634-636.

Hamilton, W. D. (1964). The genetical evolution of social behavior. I and II. J. Theor. Biol., $7: 1-52$.

Hamilton, W. D. (1967). Extraordinary sex ratios. Science, 156:477-488.

Johnstone, R. A. and Cant, M. A. (2008). Sex differences in dispersal and the evolution of helping and harming. Am. Nat., 172:318-330.

Kokko, H., Johnstone, R. A., and Clutton-Brock, T. H. (2001). The evolution of cooperative breeding through group augmentation. Proc. R. Soc. B, 268:187-196. 
Laland, K., Uller, T., Feldman, M., Sterelny, K., Müller, G. B., Moczek, A., Jablonka, E., Odling-Smee, J., Wray, G. A., Hoekstra, H., Futuyma, D. J., Lenski, R. E., Mackay, T. F. C., Schluter, D., and Strassmann, J. E. (2014). Does evolutionary biology need a rethink? Nature, 514:161-164.

Maynard Smith, J. and Price, G. R. (1973). The logic of animal conflict. Nature, 246:15-18.

Pen, I. (2006). When boys want to be girls: effects of mating system and dispersal on parent-offspring sex ratio conflict. Evol. Ecol. Res., 8:103-113.

Pen, I. and Taylor, P. D. (2005). Modelling information exchange in worker-queen conflict over sex allocation. Proc. R. Soc. B, 272:2403-2408.

Pen, I. and Weissing, F. J. (2000a). Sex-ratio optimization with helpers and the nest. Proc. R. Soc. Lond. B, 267:539-543.

Pen, I. and Weissing, F. J. (2000b). Towards a unified theory of cooperative breeding: the role of ecology and life history re-examined. Proc. R. Soc. B, 267:2411-2418.

Shaw, R. and Mohler, J. (1953). The selective significance of the sex ratio. Am. Nat., $87: 337-342$.

Stacey, P. B. and Ligon, J. D. (1991). The benefits-of-philopatry hypothesis for the evolution of cooperative breeding: variation in territory quality and group size effects. Am. Nat., 1137:831-846.

Taylor, P. D. (1989). Evolutionary stability in one-parameter models under weak selection. Theor. Popul. Biol., 36:125-143. 
Taylor, P. D. (1992). Altruism in viscous populations - an inclusive fitness model. Evol. Ecol., 6:352-356.

Taylor, P. D. (1996). Inclusive fitness and genetic models of behaviour. J. Math. Biol., $34: 654-674$.

Taylor, P. D. and Frank, S. A. (1996). How to make a kin selection model. J. Theor. Biol., $180: 27-37$.

Thompson, G. J., Hurd, P. L., and Crespi, B. J. (2013). Genes underlying altruism. Biol. Lett., 9:20130395.

Trivers, R. L. and Hare, H. (1976). Haplodiploidy and the evolution of social insects. Science, 191:249-263.

Wild, G. (2006). Sex ratios when helpers stay at the nest. Evolution, 60:2012-2022.

Wild, G. and Koykka, C. (2014). Inclusive-fitness logic of cooperative breeding with benefits of natal philopatry. Phil. Trans. R. Soc. B, 369:20130361.

Wild, G. and Taylor, P. D. (2004). Kin selection models for the co-evolution of the sex ratio and sex-specific dispersal. Evol. Ecol. Res., 6:481-502.

Wild, G. and Taylor, P. D. (2005). A kin-selection approach to the resolution of a sex-ratio conflict between mates. J. Theor. Biol., 236:126-136.

Wild, G. and Traulsen, A. (2007). The different limits of weak selection and the evolutionary dynamics of finite populations. J. Theor. Biol., 247:382-390. 
Wild, G. and West, S. A. (2009). Genomic imprinting and sex allocation. Am. Nat., 173:E1E14.

Wiley, R. H. and Rabenold, K. N. (1984). The evolution of cooperative breeding by delayed reciprocity and queuing for favorable social positions. Evolution, 38:609-621. 


\section{Figure Captions}
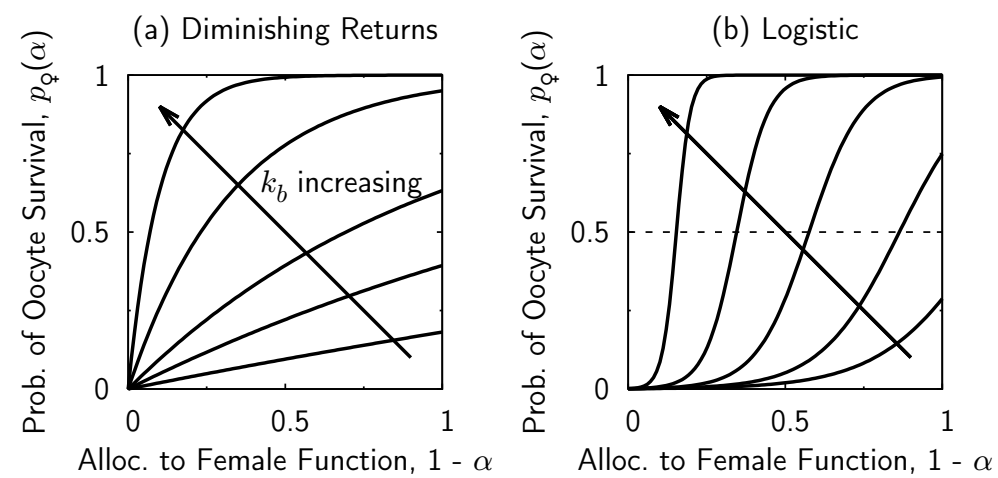

Figure 1: Comparison of the diminishing returns and logistic paradigms for reproductive functions. Recall that a breeder has $k_{b}$ resources and each unit of female reproductive function costs $c_{\varphi}$ resources. Panel (a) shows $p_{\varphi}(\alpha)$ in the diminishing returns paradigm as a function of the fraction of resources allocated towards reproduction through female function. In this panel we set $k_{b} / c_{\varphi}=0.2,0.5,1,3,10$. Panel $(\mathrm{b})$ shows $p_{\varphi}(\alpha)$ in the logistic paradigm as a function of the fraction of resources allocated towards reproduction through female function. In this panel we set $k_{b} / c_{q}=6,8,12,20,45$ with $\varepsilon_{q}=0.001$. If both reproductive functions (only $p_{\uparrow}$ is shown in this figure) are above (resp. below) the dashed line we know $\alpha^{*}$ is (resp. is not) evolutionarily stable. When the reproductive functions are less than 0.5 (below the dashed line) there are increasing rates of return on investment. When the reproductive functions are greater than 0.5 (above the dashed line) there are diminishing returns on investment. 

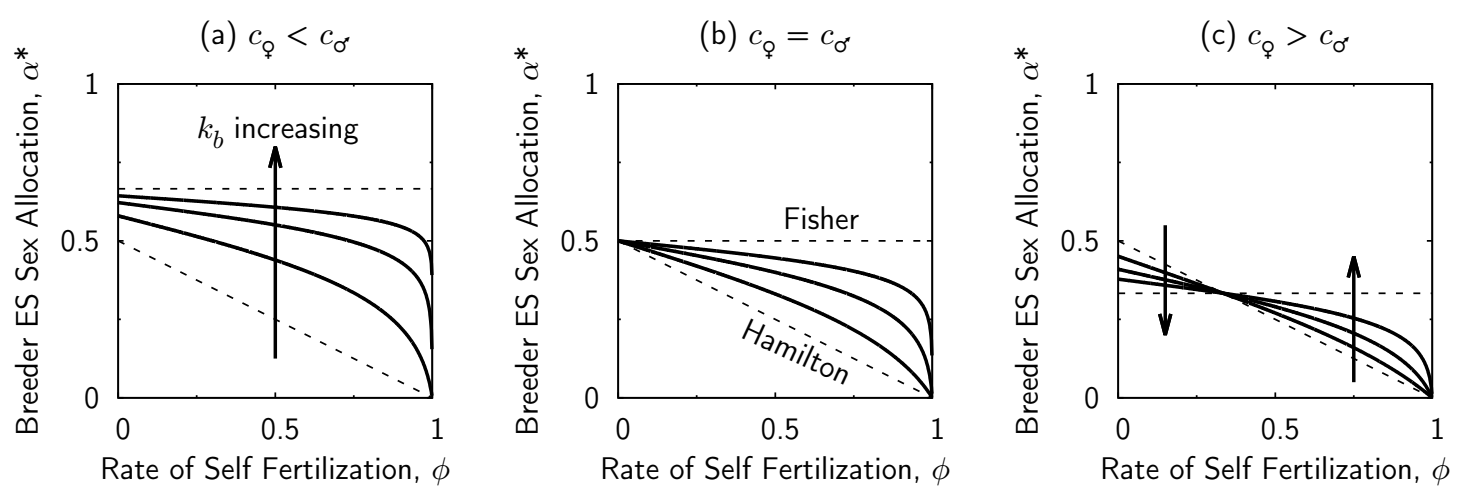

Figure 2: The ES sex-allocation strategy, $\alpha^{*}$, responds to changes in the rate of selfferlization, $\phi$, and to changes in sex-specific costs of investing in reproductive function, $c_{q}$ and $c_{\sigma^{*}}$. Panels show the ES sex-allocation strategy $\alpha^{*}$ as a function of $\phi$ when (a) investment in male function is more costly $\left(c_{Q}=0.5\right.$ and $\left.c_{\sigma^{\prime}}=1\right)$, (b) investment in male function and investment female function are equally costly (equation 4 ) $\left(c_{q}=1\right.$ and $\left.c_{\sigma^{*}}=1\right)$, and (c) investment in female function is more costly $\left(c_{\varphi}=2\right.$ and $\left.c_{\sigma^{*}}=1\right)$. The diagonal dashed curve shows the case of extreme resource limitation, $k_{b} \rightarrow 0^{+}$(Hamilton's local mate competition, equation 5). The dashed horizontal curve shows the case of extreme resource abundance, $k_{b} \rightarrow \infty$ (Fisher's equal production of sexes, equation 6). Solid curves show results for intermediate resource availability $\left(k_{b}=2,5,10\right)$. 

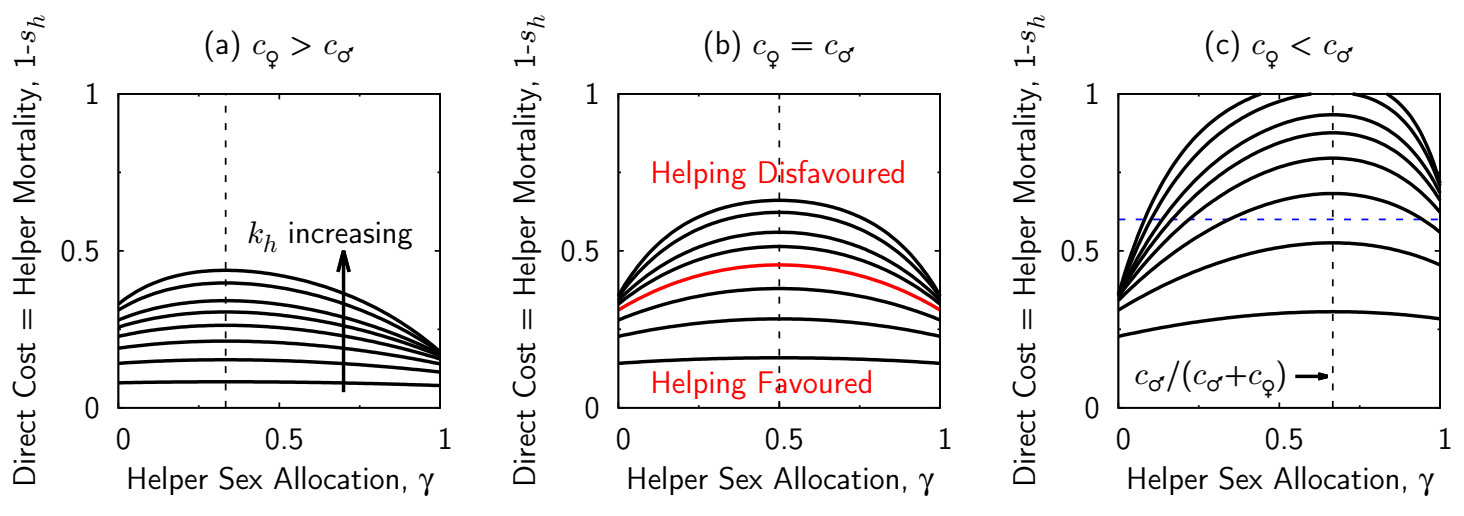

Figure 3: The critical direct cost, $1-s_{h}$, as a function of helper sex allocation strategy, $\gamma$, predicted by equation (10) when $\phi=0.2$. Note that $\alpha^{*}=0.4$ in this figure since $\alpha^{*}=(1-\phi) / 2$ in the limit as $k_{b} \rightarrow 0^{+}$. Each panel presents a range of $k_{h}$ values, beginning at 0.5 (bottommost curve) and ending at 5 (top-most curve). The values are $k_{h}=0.5,1,1.5,2,2.5,3,4,5$. Panel (a) shows the case where $c_{\varphi}=2>c_{\sigma^{\circ}}=1$, panel (b) shows the case where $c_{\varphi}=c_{\sigma^{\circ}}=1$, and panel(c) shows the case where $c_{\varphi}=0.5<c_{\sigma^{\circ}}=1$. Helping is favoured (resp. disfavoured) in the region below (resp. above) a given curve (e.g. red annotation). The vertical dashed line shows $\gamma_{\max }=c_{\sigma^{\prime}} /\left(c_{\sigma^{\prime}}+c_{q}\right)$ to highlight the qualitative change in the effect increasing gamma has on the emergence of helping: positive effect to the left, and negative effect to the right. The horizontal dashed line in blue is included to emphasize that increasing $k_{h}$ changes only quantitative features of the effect that $\gamma$ exerts on the critical direct cost, not the qualitative ones. 

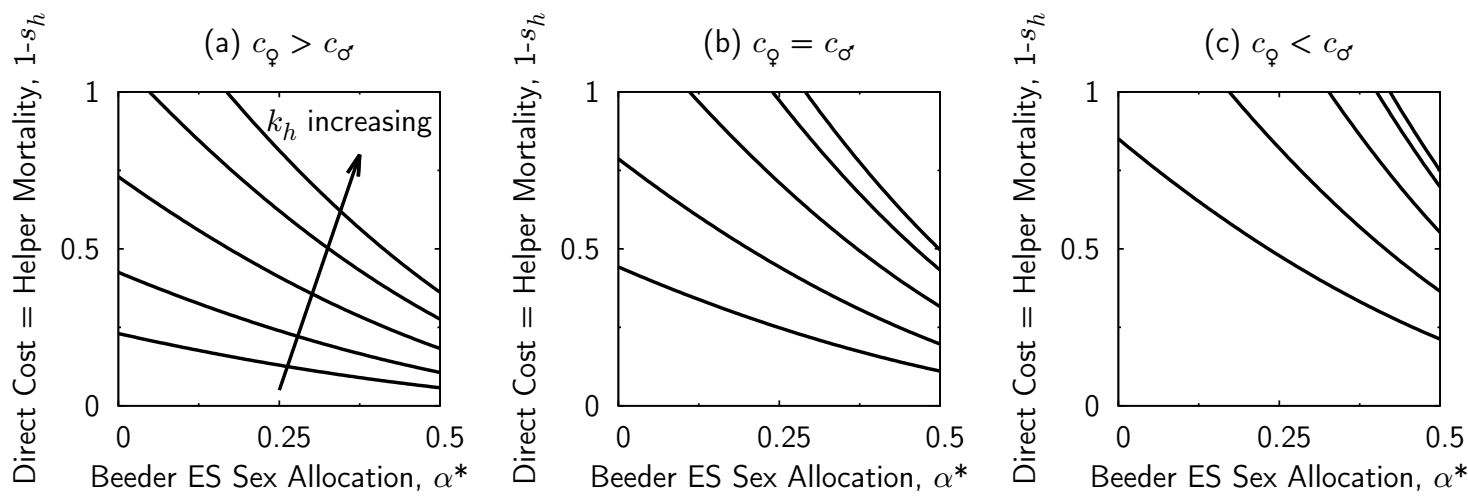

Figure 4: The critical direct cost, $1-s_{h}$, as a function of breeder ES sex allocation strategy, $\alpha^{*}=(1-\phi) / 2$, predicted by equation $(10)$. For ease of illustration we assume that helpers use the allocation strategy $\gamma=\gamma_{\max }=c_{\sigma^{\circ}} /\left(c_{\sigma^{\circ}}+c_{\varphi}\right)$. Each panel varies the amount of helper resources, $k_{h}=0.5,1,3,4,10$. Panel (a) shows the case where $c_{\varphi}=2$ and $c_{\sigma^{\prime}}=1$, panel (b) shows the case where $c_{\varphi}=1$ and $c_{\sigma^{\circ}}=1$, and panel (c) shows the case where $c_{\varphi}=0.5$ and $c_{\sigma^{\prime}}=1$. Helping is favoured (resp. disfavoured) in the region below (resp. above) a given curve. This figure demonstrates how increasing $\alpha^{*}$ - essentially decreasing relatedness - hinders the emergence of helpful behaviour for this limiting case $\left(k_{b} \rightarrow 0^{+}\right)$. For a given amount of helper resources, reducing the cost of female units of reproduction promotes the emergence of helpful behaviour. 
(a) $c_{\varphi}=0.2$

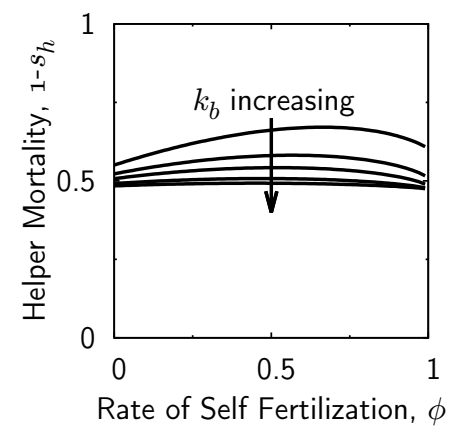

(b) $c_{\varphi}=1.0$

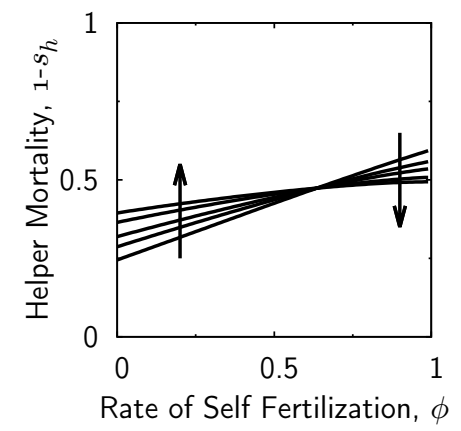

(c) $c_{\varphi}=1.8$

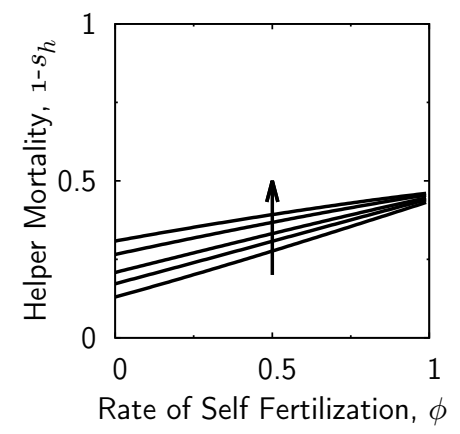

Figure 5: The critical direct cost, $1-s_{h}$, for the NTI Model as a function of the rate of self-fertilization, $\phi$ when breeders have non-vanishing levels of resource, $k_{b}$. For simplicity all plots assume $\gamma=\gamma_{\max }$, as this was sufficient to illustrate qualitative trends. Each panel presents a range of $k_{b}$ values $\left(k_{b}=0.5,0.75,1,1.5,2\right)$. Each panel also sets $s_{b}=0.9, k_{h}=1$, $c_{\sigma^{\prime}}=1$, but $c_{q}$ is varied across panels $\left((\mathrm{a}) c_{q}=0.2\right.$, (b) $c_{q}=1$, (c) $\left.c_{q}=1.8\right)$. Helping is favoured (resp. disfavoured) in the region below (resp. above) a given curve. 
(a) $c_{\text {}}=0.5$

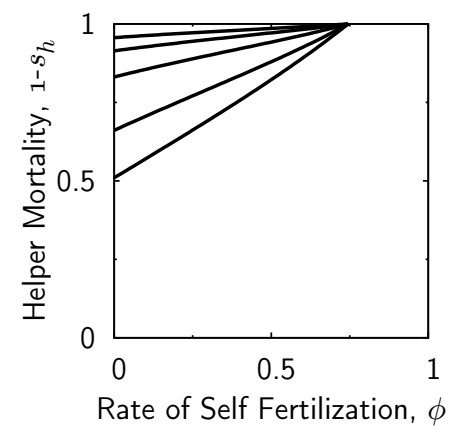

(b) $c_{\varphi}=0.68$

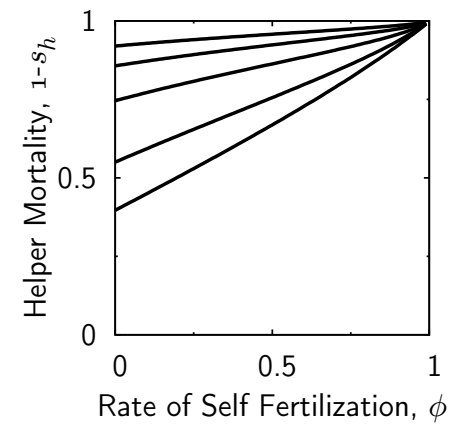

(c) $c_{\varphi}=1.3$

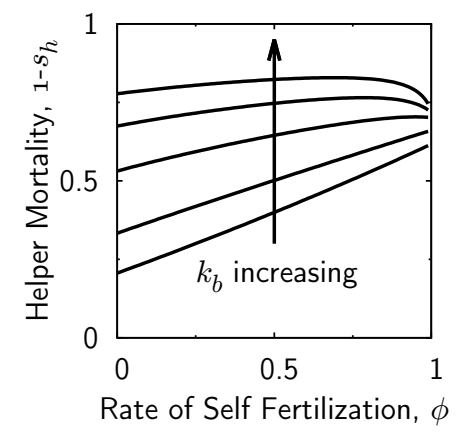

Figure 6: The critical direct cost, $1-s_{h}$, for the TI Model as a function of the rate of self-fertilization, $\phi$ when breeders have non-vanishing levels of resource, $k_{b}$. For simplicity all plots assume $\gamma=\gamma_{\max }$, as this was sufficient to illustrate qualitative trends. Each panel presents a range of $k_{b}$ values $\left(k_{b}=0.5,2,3,4\right)$. Each panel also sets $s_{b}=0.9, k_{h}=1, c_{\sigma^{*}}=1$, but $c_{q}$ is varied across panels $\left((\mathrm{a}) c_{q}=0.5\right.$, (b) $\left.c_{q}=0.68,(\mathrm{c}) c_{q}=1.3\right)$. Helping is favoured (resp. disfavoured) in the region below (resp. above) a given curve. 


\section{Appendix}

\section{A Equilibrium Solutions}

For the NTI Model, the description in the main text gives us

NTI Model:

$$
\left.\begin{array}{rl}
F(t+1) & =\left(1-\frac{1}{1+a B(t)}\right) s_{f} F(t)+p_{\phi} B(t) \\
B(t+1) & =\frac{s_{f} F(t)}{1+a B(t)}+s_{b} B(t)
\end{array}\right\}
$$

and for the TI Model, the description in the main text gives us

TI Model:

$$
\left.\begin{array}{l}
F(t+1)=\left(1-\frac{1}{1+a B(t)}\right) s_{f} F(t)+p_{\varphi} s_{b} B(t) \\
B(t+1)=\frac{s_{f} F(t)}{1+a B(t)}+\left(s_{b}+\left(1-s_{b}\right) p_{\varphi}\right) B(t) .
\end{array}\right\}
$$

The equilibrium solutions and their associated stability follow trivially from Wild and Koykka (2014) and a summary of those results are shown below. This is because the probability of producing a viable oocyte ( $p$ in their models, $p_{\odot}$ in ours) is still defined on the interval $(0,1)$. In this paper, we denote the equilibrium floater and breeder densities as $\bar{F}$ and $\bar{B}$, respectively. The table below holds true for both the NTI and TI models when the associated values for $\mathcal{R}_{0}$ are used. Recall that in the NTI model $\mathcal{R}_{0}=\frac{p_{\varphi}(\alpha)}{1-s_{b}} s_{f}$ and in the TI model $\mathcal{R}_{0}=\frac{p_{\varphi}(\alpha)}{1-s_{b}} \frac{s_{b}}{1-p_{\varphi}(\alpha)} s_{f}$.

\begin{tabular}{c|c|c|c} 
& $\bar{F}$ & $\bar{B}$ & Condition for Stability \\
\hline Trivial Equilibrium & 0 & 0 & $\mathcal{R}_{0}<1$ \\
\hline Positive Equilibrium & $\left(\frac{p_{\odot}(\alpha)}{1-s_{b}}-1\right) \frac{1-s_{b}}{1-s_{f}} \frac{\mathcal{R}_{0}-1}{a\left(1-s_{f}\right)}$ & $\frac{\mathcal{R}_{0}-1}{a\left(1-s_{f}\right)}$ & $\mathcal{R}_{0}>1$
\end{tabular}




\section{B Coefficients of Relatedness}

Throughout the main text we use coefficients of relatedness that were calculated using a coefficient of consanguinity argument. The first step in this argument is to determine the coefficient of inbreeding, which we will call $\vartheta$. This is the probability both alleles in an individual are identical by descent. To find the coefficient of inbreeding in the next season we use a simple replacement argument. The subscript $n$ denotes the current season and the subscript $n+1$ denotes the next season. With probability $\phi$ a breeder self-fertilizes and with equal probability the offspring inherits two of the same allele, which are clearly identical by descent or different alleles, which are identical by descent with probability $\vartheta_{n}$. When a breeder outcrosses the alleles in the offspring are never identical by descent by assumption. Mathematically,

$$
\vartheta_{n+1}=\phi \frac{1+\vartheta_{n}}{2}
$$

We expect $\vartheta_{n+1}=\vartheta_{n}=\bar{\vartheta}$ at equilibrium, and conclude the equilibrium coefficient of inbreeding is,

$$
\bar{\vartheta}=\frac{\phi}{2-\phi}
$$

The next part of this argument derives the desired coefficient of consanguinities (CC). This is the blood relatedness between two individuals. To determine the CC between an individual and itself we will choose one of their two alleles (we assume individuals are diploid) at random, replace it, and choose again at random. Half of the time we will choose the same allele, which is clearly identical by descent. Half of the time we will choose different alleles, 
which are identical by descent with probability $\bar{\vartheta}$. Mathematically,

$$
\frac{1+\bar{\vartheta}}{2}=\frac{1}{2-\phi}
$$

We had to calculate the CC between an individual and itself because relatedness terms are expressed as a ratio of CCs. For example, the relatedness between an individual and its mother is the $\mathrm{CC}$ between an individual and its mother divided by the $\mathrm{CC}$ between an individual and itself.

Now we will calculate the relatedness between a breeder and its offspring produced through male function as a product of outcrossing, $r_{b, \sigma^{*}}$. A quarter of the time we choose the same allele in the paternal parent and offspring, which is clearly identical by descent. A quarter of the time we choose different alleles (but still from the paternal parent), which are identical by descent with probability $\bar{\vartheta}$. The CC between a breeder and its offspring produced through female function is,

$$
\frac{1+\bar{\vartheta}}{4}=\frac{1}{2} \frac{1}{2-\phi}
$$

Recall that relatedness is calculated as the ratio of CCs. Following this logic, we must divide the above equation by the $\mathrm{CC}$ between an individual and itself (equation (B.1) ). The coefficient of relatedness between an offspring and its paternal parent is,

$$
r_{b, 0^{*}}=\frac{1}{2}
$$

Next, we will calculate the relatedness between a breeder and its offspring produced through female function, $r_{b, q}$. The CC between a breeder and its offspring produced through female function is the the sum of two probabilities. The first occurs with probability $\phi$, and is when the offspring is a product of self-fertilization. Half of the time we choose the same 
allele, which is clearly identical by descent, and half of the time we choose a different allele, which is identical by descent with probability $\bar{\vartheta}$. The second occurs with probability $1-\phi$, and is when the offspring is a product of outcrossing. A quarter of the time we choose the same allele, which is clearly identical by descent. A quarter of the time we choose a different allele (but still from the maternal parent), and it is identical by descent with probability $\bar{\vartheta}$. The CC between the maternal parent and an offspring produced through female function is,

$$
\phi \frac{1+\bar{\vartheta}}{2}+(1-\phi) \frac{1+\bar{\vartheta}}{4}=\frac{1}{2} \frac{1+\phi}{2-\phi} .
$$

Once again, we must divide the above equation by the $\mathrm{CC}$ between an individual and itself (equation (B.1) ). This gives the relatedness between an individual and its maternal parent,

$$
r_{b, q}=\frac{1+\phi}{2}
$$

We can express the relatedness between siblings with a common parent as product of $r_{b, q}$ and $r_{b, 0^{*}}$. The relatedness between siblings with the same maternal parent (i.e., each individual has relatedness $r_{b, q}$ with this parent) is,

$$
r_{h, q}=r_{b, q} r_{b, q}=\left(\frac{1+\phi}{2}\right)^{2}
$$

The relatedness between siblings with a common parent where one is produced through female function and the other through male function (i.e., one individual has relatedness $r_{b, \circ}$ and the other has relatedness $r_{b, 0^{*}}$ with this parent) is,

$$
r_{h, \sigma^{*}}=r_{b, q} r_{b, \sigma^{*}}=\frac{1+\phi}{4}
$$




\section{Mutant Fitness for Sex Allocation}

Since individuals are diploid and can self-fertilize, offspring may receive either zero, one, or two copies of the mutant allele. These individuals will be referred to as homozygous resident, heterozygous, and homozygous mutant, respectively. As mentioned in the main text, this mutation alters an individual's sex allocation resulting in a change to the fraction of resources invested in male components of breeder fitness, $\alpha$. The change in investment is proportional to the number of mutant alleles an individual has. We assume additive genetic effects. We define an individual's sex allocation as $\alpha_{i}=\alpha_{0}+i \delta$, where $i=0,1,2$ are the number of mutant alleles an individual has and $\delta$ is the phenotypic deviation per mutant allele. The phenotypic deviation is assumed to be small (weak selection) and can be either positive or negative. Following the population dynamics described in the main text, the mutant population can be described by four compartments:

- the density of heterozygous floaters, $u_{1}$;

- the density of homozygous mutant floaters, $u_{2}$;

- the density of heterozygous breeders, $v_{1}$;

- the density of homozygous mutant breeders, $v_{2}$.

\section{C.1 Model I: No Territory Inheritance (NTI)}

To carry out this invasion analysis we follow the method presented in Wild and Koykka (2014). When there is no territory inheritance in the resident population, the dynamics of 
the mutant population is described by,

$$
\left[\begin{array}{l}
u_{1}^{\prime} \\
u_{2}^{\prime} \\
v_{1}^{\prime} \\
v_{2}^{\prime}
\end{array}\right]=\underbrace{\left[\begin{array}{cccc}
\mathcal{J}_{11} & 0 & \mathcal{J}_{13} & \mathcal{J}_{14} \\
0 & \mathcal{J}_{22} & \mathcal{J}_{23} & \mathcal{J}_{24} \\
\mathcal{J}_{31} & 0 & \mathcal{J}_{33} & 0 \\
0 & \mathcal{J}_{42} & 0 & \mathcal{J}_{44}
\end{array}\right]}_{\equiv \mathcal{J}}\left[\begin{array}{l}
u_{1} \\
u_{2} \\
v_{1} \\
v_{2}
\end{array}\right],
$$

where primes denote the next time step. The non-zero entries of $\mathcal{J}$ are found in Table 1 . A linear stability analysis is used to find the conditions for invasion. Invasion is determined by the magnitude of the largest eigenvalue, $\lambda$, and can be interpreted biologically as the long term geometric growth rate. If $\lambda<1$ the mutant, on average, is unable to replace itself and is eliminated. However, if $\lambda>1$ the mutant, on average, does better than replace itself and as a result, the invasion is successful. If $\lambda=1$ the mutant is neither eliminated nor favoured by selection. To verify if our model is biologically reasonable, we calculate the largest eigenvalue when there is no phenotypic change in the population (i.e., the mutant uses the same strategy as a normal individual, $\delta=0)$ using a computer algebra package. As expected, we find $\lambda=1$ in this case.

A first order Taylor expansion is used to approximate the long term geometric growth rate. This is valid because of our weak selection assumption ( $\delta$ is small). We find, $\lambda \approx 1-\left.\delta \frac{d \lambda}{d \delta}\right|_{\delta=0}$, plus terms of $O\left(\delta^{2}\right)$, which we ignore due to our weak selection assumption. Consequently, invasion occurs when $\left.\frac{d \lambda}{d \delta}\right|_{\delta=0}$ and $\delta$ are the opposite sign. To find $\left.\frac{d \lambda}{d \delta}\right|_{\delta=0}$ we implicitly differentiate the characteristic equation of $\mathcal{J}$ using a computer algebra package and set $\delta=0$ and $\lambda=1$. When we use the diminishing returns paradigm the selection gradient for 
the geometric growth rate is,

$$
\begin{aligned}
& \left.\frac{d \lambda}{d \delta}\right|_{\delta=0}=\frac{2}{2-\phi} \frac{\left(1-s_{b}\right)\left(1-s_{f}\right) p_{\varphi}\left(\alpha_{0}\right)}{\left(2-s_{f}-s_{b}\right) p_{\varphi}\left(\alpha_{0}\right)-\left(1-s_{b}\right)^{2}}
\end{aligned}
$$

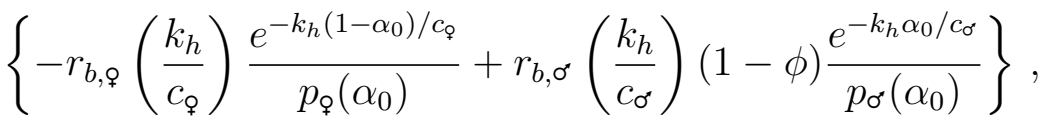

and is

$$
\begin{aligned}
& \left.\frac{d \lambda}{d \delta}\right|_{\delta=0}=\frac{2}{2-\phi} \frac{\left(1-s_{b}\right)\left(1-s_{f}\right) p_{\varphi}\left(\alpha_{0}\right)}{\left(2-s_{f}-s_{b}\right) p_{\varphi}\left(\alpha_{0}\right)-\left(1-s_{b}\right)^{2}} \\
& \left\{-r_{b, q} \frac{k_{b}}{c_{q}}\left(1-p_{\varphi}\left(\alpha_{0}\right)\right)+r_{b, \sigma^{\prime}}(1-\phi) \frac{k_{b}}{c_{\sigma^{\prime \prime}}}\left(1-p_{\sigma^{*}}\left(\alpha_{0}\right)\right)\right\},
\end{aligned}
$$

in the logistic paradigm. The term inside the curled brackets determines the sign of the selection gradient. This is because the term outside the curled brackets is always positive. Trivially, $\left(1-s_{b}\right)\left(1-s_{f}\right) p_{\varphi}\left(\alpha^{*}\right)>0$ and $\frac{2}{2-\phi}>0$, but $\left(2-s_{f}-s_{b}\right) p_{\varphi}\left(\alpha_{0}\right)-\left(1-s_{b}\right)^{2}$ requires some investigation. We claim,

$$
\left(2-s_{f}-s_{b}\right) p_{\wp}\left(\alpha_{0}\right)-\left(1-s_{b}\right)^{2}
$$

is always positive. The above expression decreases as $s_{f}$ is increased. If this function is positive when $s_{f}=1$ all other cases $\left(0<s_{f}<1\right)$ will follow trivially. After making this substitution we obtain,

$$
\left(1-s_{b}\right) p_{\varphi}\left(\alpha_{0}\right)-\left(1-s_{b}\right)^{2}=\left(1-s_{b}\right)\left(p_{\varphi}\left(\alpha_{0}\right)+s_{b}-1\right)
$$

For positivity, we require $\left(p_{\varphi}\left(\alpha_{0}\right)+s_{b}-1\right)>0$. By assumption, $\mathcal{R}_{0}=\frac{p_{\varphi}\left(\alpha_{0}\right) s_{f}}{1-s_{b}}>1$ so we know $p_{\varphi}\left(\alpha_{0}\right)>p_{\varphi}\left(\alpha_{0}\right) s_{f}>1-s_{b}$, which easily rearranges to $p_{\varphi}\left(\alpha_{0}\right)+s_{b}-1>0$. This proves the expression outside the curled brackets in equation (C.2 is always positive. As a result, the mutant invades when the term inside the curled braces and the phenotypic deviation, $\delta$, are the opposite sign. 


\section{C.2 Model II: Territory Inheritance (TI)}

When there is no territory inheritance in the resident population the dynamics of the mutant population is described by,

$$
\left[\begin{array}{l}
u_{1}^{\prime} \\
u_{2}^{\prime} \\
v_{1}^{\prime} \\
v_{2}^{\prime}
\end{array}\right]=\underbrace{\left[\begin{array}{cccc}
\mathcal{K}_{11} & 0 & \mathcal{K}_{13} & \mathcal{K}_{14} \\
0 & \mathcal{K}_{22} & \mathcal{K}_{23} & \mathcal{K}_{24} \\
\mathcal{K}_{31} & 0 & \mathcal{K}_{33} & \mathcal{K}_{34} \\
0 & \mathcal{K}_{42} & \mathcal{K}_{43} & \mathcal{K}_{44}
\end{array}\right]}_{\equiv \mathcal{K}}\left[\begin{array}{l}
u_{1} \\
u_{2} \\
v_{1} \\
v_{2}
\end{array}\right],
$$

where prime denotes the next time step. The non-zero entries of $\mathcal{K}$ can be found in Table 2 . Following the previous section, we calculate the largest eigenvalue using a computer algebra package when there is no phenotypic change in the population (i.e., the mutant uses the same strategy as a normal individual) as a check. We find $\lambda=1$ in this case, which again is expected.

We use the same approximation for the long-term geometric growth rate of the mutant

population, $\lambda \approx 1-\left.\delta \frac{d \lambda}{d \delta}\right|_{\delta=0}$, and recall that invasion happens when $\left.\frac{d \lambda}{d \delta}\right|_{\delta=0}$ and $\delta$ are the opposite sign. Similar to the NTI model, we implicitly differentiate the characteristic equation of $\mathcal{K}$ using a computer algebra package and set $\delta=0$ and $\lambda=1$. In the diminishing returns paradigm the expression for the selection gradient of the long term geometric growth rate is,

$$
\begin{aligned}
& \left.\frac{d \lambda}{d \delta}\right|_{\delta=0}=\frac{2}{2-\phi} \frac{\left(1-s_{b}\right)\left(1-s_{f}\right) s_{b} p_{\varphi}\left(\alpha_{0}\right)}{\left(s_{b}^{2}-s_{b}\left(2+s_{f}\right)+2\right) p_{\varphi}\left(\alpha_{0}\right)-\left(1-s_{b}\right)^{2}-\left(1-s_{b}\right) p_{\varphi}\left(\alpha_{0}\right)^{2}}
\end{aligned}
$$

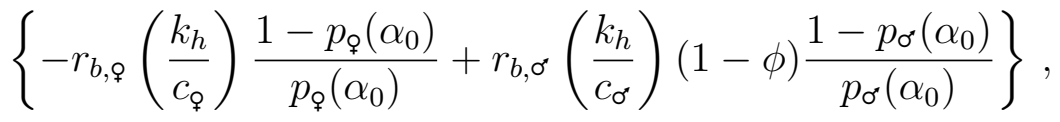


and is

$$
\begin{aligned}
& \left.\frac{d \lambda}{d \delta}\right|_{\delta=0}=\frac{2}{2-\phi} \frac{\left(1-s_{b}\right)\left(1-s_{f}\right) s_{b} p_{\varphi}\left(\alpha_{0}\right)}{\left(s_{b}^{2}-s_{b}\left(2+s_{f}\right)+2\right) p_{\varphi}\left(\alpha_{0}\right)-\left(1-s_{b}\right)^{2}-\left(1-s_{b}\right) p_{\varphi}\left(\alpha_{0}\right)^{2}}
\end{aligned}
$$

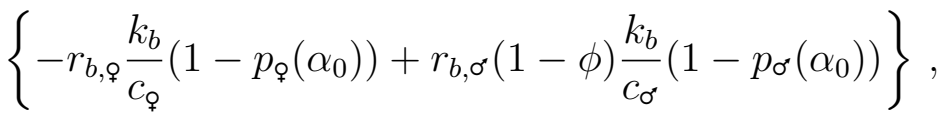

in the logistic paradigm. The term inside the curled braces determines the sign of the selection gradient. This is because we claim the term in the denominator outside of the curled braces is always positive. Once again, it is easy to see $\left(1-s_{b}\right)\left(1-s_{f}\right) s_{b} p q\left(\alpha_{0}\right)>0$ and $\frac{2}{2-\phi}>0$, but the sign of the denominator is unclear. We must show,

$$
\left(s_{b}^{2}-s_{b}\left(2+s_{f}\right)+2\right) p_{\varphi}\left(\alpha_{0}\right)-\left(1-s_{b}\right)^{2}-\left(1-s_{b}\right) p_{\varphi}\left(\alpha_{0}\right)^{2}>0 .
$$

The LHS of the inequality above is a decreasing function of $s_{f}$ and we are interested in its value when $s_{f}=1$. If the above inequality holds true in this case, it will trivially hold for all other cases when $0<s_{f}<1$. After making this substitution we must now show,

$$
\left(s_{b}^{2}-3 s_{b}+2\right) p_{\varphi}\left(\alpha_{0}\right)-\left(1-s_{b}\right)^{2}-\left(1-s_{b}\right) p_{\varphi}\left(\alpha_{0}\right)^{2}=\left(1-s_{b}\right)\left(1-p_{\varphi}\left(\alpha_{0}\right)\right)\left(p_{\uparrow}\left(\alpha_{0}\right)+s_{b}-1\right)>0 \text {. }
$$

Similar to the NTI model, the proof relies on $\left(p_{\varphi}\left(\alpha_{0}\right)+s_{b}-1\right)$ being positive, which is again true because of the assumption that $\mathcal{R}_{0}=\frac{p_{\varphi}\left(\alpha_{0}\right) s_{f}}{1-s_{b}} \frac{s_{b}}{1-p_{\varphi}\left(\alpha_{0}\right)}>1$. We find $p_{\varphi}\left(\alpha_{0}\right) s_{b}>$ $p_{\varphi}\left(\alpha_{0}\right) s_{b} s_{f}>\left(1-s_{b}\right)\left(1-p_{\varphi}\left(\alpha_{0}\right)\right)$, which easily rearranges to $p_{\varphi}\left(\alpha_{0}\right)+s_{b}-1>0$, as required. As a result, we conclude that the mutant invades whenever the term inside the curled braces and the phenotypic deviation, $\delta$, are the opposite sign. 


\section{Classifying Strategies - Diminishing Returns}

\section{D.1 Evolutionary Equilibrium (EE)}

The condition for the evolutionary equilibrium is $\left.\frac{d \lambda}{d \delta}\right|_{\delta=0}=0$, and we solve for $\alpha_{0}=\alpha^{*}$. Based on equation (C.2) and (C.4) we can easily see that these conditions are the same as the one found in the main text. For a sex allocation to be an evolutionary equilibrium it must satisfy,

$$
-r_{b, q}\left(\frac{k_{b}}{c_{q}}\right) \frac{1-p_{\odot}\left(\alpha^{*}\right)}{p_{\varphi}\left(\alpha^{*}\right)}+r_{b, \sigma^{*}}(1-\phi)\left(\frac{k_{b}}{c_{\sigma^{*}}}\right) \frac{1-p_{\sigma^{*}}\left(\alpha^{*}\right)}{p_{\sigma^{*}}\left(\alpha^{*}\right)}=0 .
$$

We require $\phi \in[0,1)$. The above equation can be solved explicitly for $\alpha^{*}$ when the costs of reproduction are equal $\left(c_{q}=c_{\sigma^{*}}=c\right)$, and numerically for all other cases. This expression is,

$$
\alpha^{*}=\frac{c}{k_{b}} \log \left(\frac{\phi+\sqrt{\phi^{2}+\left(1-\phi^{2}\right) e^{k_{b} / c}}}{1+\phi}\right) \text {. }
$$

\section{D.2 Evolutionarily Stable (ES)}

To determine whether an EE strategy, $\alpha^{*}$, is ES we use a derivative condition from Courteau \& Lessard (2000). Since we assume the genetic effects are additive we know that $\alpha_{2}=$ $2 \alpha_{1}-\alpha_{0}$, which is an essential redefinition to make use of the results from their paper.

Biologically, when a strategy is ES it means that all other strategies provide a lower fitness, and as a result cannot invade. The strategy $\alpha^{*}$ is ES when

$$
\left.\frac{\partial^{2} f_{\mathrm{NTI}}}{\partial \alpha_{1}^{2}}\right|_{\alpha_{1}=\alpha_{0}=\alpha^{*}}>0, \quad \text { where } \quad f_{\mathrm{NTI}}=\operatorname{det}\left(\mathbf{I}-\mathcal{J}\left(\alpha_{0}, \alpha_{1}\right)\right),
$$


for the NTI model and

$$
\left.\frac{\partial^{2} f_{\mathrm{TI}}}{\partial \alpha_{1}^{2}}\right|_{\alpha_{1}=\alpha_{0}=\alpha^{*}}>0, \quad \text { where } \quad f_{\mathrm{TI}}=\operatorname{det}\left(\mathbf{I}-\mathcal{K}\left(\alpha_{0}, \alpha_{1}\right)\right),
$$

for the TI model, where $\mathbf{I}$ is the identity matrix. The invasion matrices $\mathcal{J}$ and $\mathcal{K}$ are discussed in equations (C.1) and (C.2), respectively.

\section{D.2.1 NTI Model}

We use a computer algebra package to check the derivative condition in equation (D.2). To simplify the output we make use of the fact that all candidate ES strategies are EE. With this simplification, the resulting condition is

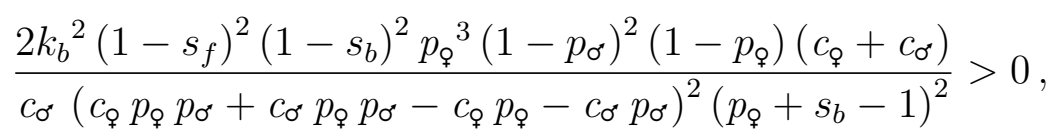

which is clearly always positive. Thus, when we assume diminishing returns, $\alpha^{*}$ is always ES in the TI model.

\section{D.2.2 TI Model}

Similar to the NTI model, we use a computer algebra package to check the derivative condition in equation (D.3). Again, we simplify the output by knowing that all candidate ES strategies are also EE. The resulting condition is

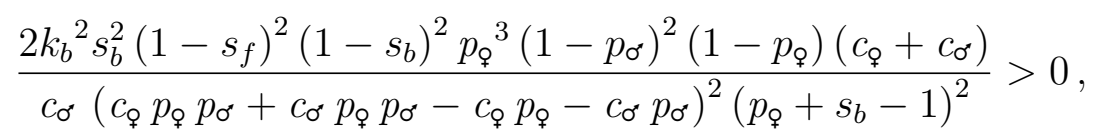

which, again, is always positive. Thus, by assuming diminishing returns, $\alpha^{*}$ is always ES in the NTI model. 


\section{D.3 Convergence Stable (CS)}

To determine whether the EE strategy, $\alpha^{*}$, is CS we use the derivative condition

$$
\frac{d}{d \alpha}\left[\left.\frac{\partial W}{\partial \beta}\right|_{\beta=\alpha}\right]_{\alpha=\alpha^{*}}<0
$$

discussed in Taylor (1996). When a strategy is CS it means that the direction from which selection approaches the EE strategy does not matter. This calculation is the same for both the NTI and TI model. Note that when we assume diminishing returns for the reproductive functions

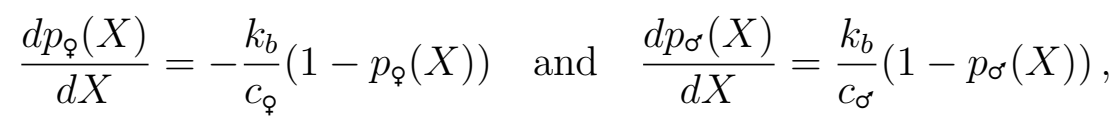

where $X=\alpha, \beta$. We begin with the expression for fitness, $W$, found in the manuscript. The proof for CS is as follows:

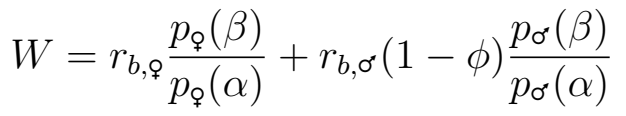

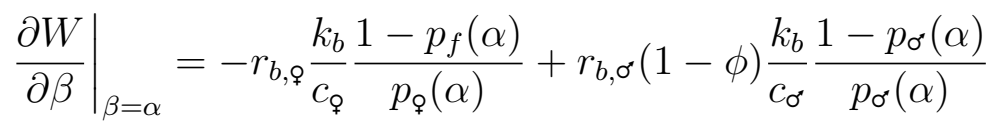

$$
\begin{aligned}
& \left.\frac{\partial W}{\partial \beta}\right|_{\beta=\alpha}=-r_{b, q} \frac{k_{b}}{c_{\varphi}}\left(\frac{1}{p_{\varphi}(\alpha)}-1\right)+r_{b, \sigma^{*}}(1-\phi) \frac{k_{b}}{c_{\sigma^{*}}}\left(\frac{1}{p_{\sigma^{*}}(\alpha)}-1\right) \\
& \frac{d}{d \alpha}\left[\left.\frac{\partial W}{\partial \beta}\right|_{\beta=\alpha}\right]_{\alpha=\alpha^{*}}=-r_{b, q} \frac{k_{b}^{2}}{c_{\varphi}^{2}} \frac{1-p_{\phi}\left(\alpha^{*}\right)}{p_{\varphi}^{2}\left(\alpha^{*}\right)}-r_{b, \sigma^{*}}(1-\phi) \frac{k_{b}^{2}}{c_{\sigma^{*}}^{2}} \frac{1-p_{\text {ơ }^{*}}\left(\alpha^{*}\right)}{p_{\sigma^{\prime}}^{2}\left(\alpha^{*}\right)}<0
\end{aligned}
$$

Thus, $\alpha^{*}$ is always CS in both the NTI and TI models when we assume diminishing returns for the reproductive functions.

\section{D.4 Limited resources $\left(k_{b} \rightarrow 0^{+}\right)$}

The ES sex allocation in these models is Hamilton's Local Mate Competition (LMC) result when breeders are extremely resource limited. We use the approximation $p_{q} \approx k_{b}(1-$ 
$x) / c_{q}+O\left(\left(-k_{b}(1-x) / c_{\varphi}\right)^{2}\right)$ and $p_{\sigma^{*}} \approx k_{b} x / c_{\sigma^{*}}+O\left(\left(-k_{b} x / c_{\sigma^{*}}\right)^{2}\right)$, which are first order Taylor expansions. Substituting these approximations into the corresponding fitness function (in the main text), we find,

$$
\begin{aligned}
W(\beta, \alpha) \approx W_{\text {approx }}(\beta, \alpha)=r_{b, q} \frac{k_{b}(1-\beta) / c_{q}+O\left(\left(-k_{b}(1-\beta) / c_{q}\right)^{2}\right)}{k_{b}(1-\alpha) / c_{q}+O\left(\left(-k_{b}(1-\alpha) / c_{q}\right)^{2}\right)} & \\
& +r_{b, \sigma^{\circ}}(1-\phi) \frac{k_{b} \beta / c_{\sigma^{\circ}}+O\left(\left(-k_{b} \beta / c_{\sigma^{\prime}}\right)^{2}\right)}{k_{b} \alpha / c_{\sigma^{\circ}}+O\left(\left(-k_{b} \alpha / c_{\sigma^{*}}\right)^{2}\right)} .
\end{aligned}
$$

In the limit as $k_{b} \rightarrow 0^{+}$the higher order terms approach zero. After taking this limit the expression becomes,

$$
W_{\text {approx }}(\beta, \alpha)=r_{b, q} \frac{1-\beta}{1-\alpha}+r_{b, \sigma^{\prime}}(1-\phi) \frac{\beta}{\alpha} .
$$

We differentiate the above equation with respect to $\beta$ to find the condition for a sex allocation to be an evolutionary equilibrium in this limiting case. This condition is,

$$
\left.\frac{\partial W_{\text {approx }}(\beta, \alpha)}{\partial \beta}\right|_{\beta=\alpha=\alpha^{*}}=-r_{b, \$} \frac{1}{1-\alpha^{*}}+r_{b, \sigma^{*}}(1-\phi) \frac{1}{\alpha^{*}}=0 .
$$

Recall $r_{b, q}=(1+\phi) / 2$ and $r_{b, \sigma^{*}}=1 / 2$. Solving the above derivative condition for $\alpha^{*}$ we find,

$$
\alpha^{*}=\frac{1-\phi}{2}
$$

\section{D.5 Unlimited resources $\left(k_{b} \rightarrow \infty\right)$}

We show that in the limit as $k_{b} \rightarrow \infty$ (breeders become resource unlimited) the evolutionary equilibrium breeder sex allocation, $\alpha^{*}$, reduces to a simple expression involving sex specific costs of reproduction. Mathematically, we recover,

$$
\alpha^{*}=\frac{c_{\sigma^{*}}}{c_{\sigma^{*}}+c_{\varphi}} .
$$


Before we take this limit we must first do some rearrangement. For a breeder sex allocation to be an evolutionary equilibrium, $\alpha=\alpha^{*}$, it must satisfy,

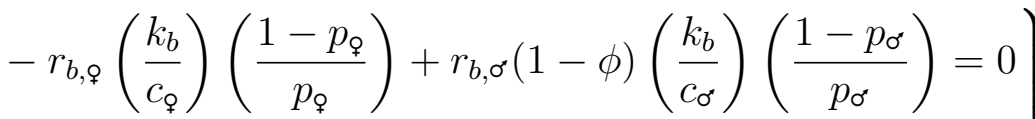

$$
\begin{aligned}
& \Longrightarrow-r_{b, q}\left(\frac{1}{c_{q}}\right)\left(\frac{1-p_{\varphi}}{p_{\varphi}}\right)+r_{b, \sigma^{\prime}}(1-\phi)\left(\frac{1}{c_{\sigma^{\prime}}}\right)\left(\frac{1-p_{\sigma^{\prime}}}{p_{\sigma^{\prime}}}\right)=0
\end{aligned}
$$

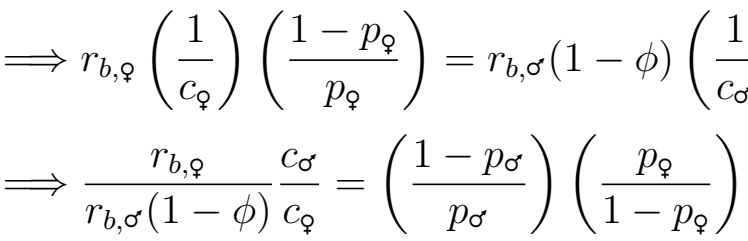

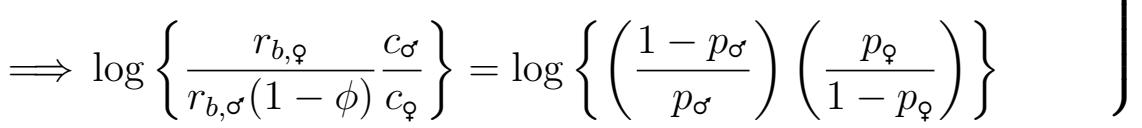

Since $c_{\varphi}, c_{\sigma^{*}}, r_{b, q}$, and $r_{b, \sigma^{*}}$ are all positive, $p_{\varphi}$ and $p_{\sigma^{*}}$ are defined on the interval $(0,1)$, and $\phi$ is defined on the interval $[0,1)$, the above step is justified. For the subsequent steps of this proof let $a=\log \left\{\frac{r_{b, q}}{r_{b, \sigma^{\circ}}(1-\phi)} \frac{c_{\sigma^{*}}}{c_{\odot}}\right\}$. Note that this expression does not depend on the amount of breeder resources, $k_{b}$, since $r_{b, q}=(1+\phi) / 2$ and $r_{b, \sigma^{*}}=1 / 2$. Recall that when $\alpha=\alpha^{*}, p_{\odot}=1-\exp \left\{-k_{b}\left(1-\alpha^{*}\right) / c_{\varphi}\right\}$ and $p_{\sigma^{*}}=1-\exp \left\{-k_{b} \alpha^{*} / c_{\sigma^{*}}\right\}$. From the above equation we know that

$$
\begin{aligned}
a & =\log \left\{\left(\frac{1-p_{\sigma^{*}}}{p_{\sigma^{*}}}\right)\left(\frac{p_{\odot}}{1-p_{\varphi}}\right)\right\} \\
& =\log \left\{1-p_{\sigma^{*}}\right\}-\log \left\{p_{\sigma^{*}}\right\}+\log \left\{p_{\varphi}\right\}-\log \left\{1-p_{\phi}\right\} \\
& =\log \left\{e^{-k_{b} \alpha^{*} / c_{\sigma^{*}}}\right\}-\log \left\{1-e^{-k_{b} \alpha^{*} / c_{\sigma^{*}}}\right\}+\log \left\{1-e^{-k_{b}\left(1-\alpha^{*}\right) / c_{\varphi}}\right\}-\log \left\{e^{-k_{b}\left(1-\alpha^{*}\right) / c_{\varphi}}\right\} \\
& =-\frac{k_{b} \alpha^{*}}{c_{\sigma^{*}}}-\log \left\{1-e^{-k_{b} \alpha^{*} / c_{\sigma^{*}}}\right\}+\log \left\{1-e^{-k_{b}\left(1-\alpha^{*}\right) / c_{\varphi}}\right\}+\frac{k_{b}\left(1-\alpha^{*}\right)}{c_{\varphi}}
\end{aligned}
$$

With some rearrangement we find,

$$
\alpha^{*}=\frac{c_{\sigma^{*}}}{c_{\sigma^{*}}+c_{\varphi}}+\frac{1}{k_{b}}\left(\frac{c_{\sigma^{*}} c_{q}}{c_{\sigma^{*}}+c_{\varphi}}\right)\left(\log \left\{1-e^{-k_{b}\left(1-\alpha^{*}\right) / c_{\varphi}}\right\}-\log \left\{1-e^{-k_{b} \alpha^{*} / c_{\sigma^{*}}}\right\}-a\right) .
$$

The prior steps isolated the terms that do not shrink as $k_{b}$ becomes large, which allows 
us to take the limit as $k_{b} \rightarrow \infty$ and recover important results. The limit is

$$
\begin{aligned}
\lim _{k_{b} \rightarrow \infty} \alpha^{*}=\lim _{k_{b} \rightarrow \infty} \frac{c_{\sigma^{*}}}{c_{\sigma^{*}}+c_{\varphi}} & \\
& +\lim _{k_{b} \rightarrow \infty} \frac{1}{k_{b}} \frac{c_{\sigma^{*}} c_{\varphi}}{c_{\sigma^{*}}+c_{\varphi}}\left(\log \left\{1-e^{-k_{b}\left(1-\alpha^{*}\right) / c_{\varphi}}\right\}-\log \left\{1-e^{-k_{b} \alpha^{*} / c_{\sigma}}\right\}-a\right) .
\end{aligned}
$$

We substitute the value of $a$ back into the above equation to easily take all limits. The resulting limit is

$$
\begin{array}{r}
\lim _{k_{b} \rightarrow \infty} \alpha^{*}=\lim _{k_{b} \rightarrow \infty} \frac{c_{\sigma^{*}}}{c_{\sigma^{*}}+c_{\varphi}}+\lim _{k_{b} \rightarrow \infty} \frac{1}{k_{b}} \frac{c_{\sigma^{*}} c_{\varphi}}{c_{\sigma^{*}}+c_{\varphi}}\left(\log \left\{1-e^{-k_{b}\left(1-\alpha^{*}\right) / c_{\varphi}}\right\}-\log \left\{1-e^{-k_{b} \alpha^{*} / c_{\sigma^{*}}}\right\}\right) \\
-\lim _{k_{b} \rightarrow \infty} \frac{1}{k_{b}} \frac{c_{\sigma^{*}} c_{\varphi}}{c_{\sigma^{*}}+c_{\varphi}}\left(\log \left\{\frac{r_{b, \phi}}{r_{b, \sigma^{*}}(1-\phi)} \frac{c_{\sigma^{*}}}{c_{\varphi}}\right\}\right) .
\end{array}
$$

Both logarithms that depend on $k_{b}$ approach 0 , and the denominator of the last two limits approach $\infty$. Finally, as breeders become resource unlimited, we find

$$
\alpha^{*}=\frac{c_{\sigma^{*}}}{c_{\sigma^{*}}+c_{\propto}} \text {. }
$$

\section{E Classifying Strategies - Logistic}

\section{E.1 Evolutionary Equilibrium (EE)}

The EE sex allocation, $\alpha^{*}$, occurs when

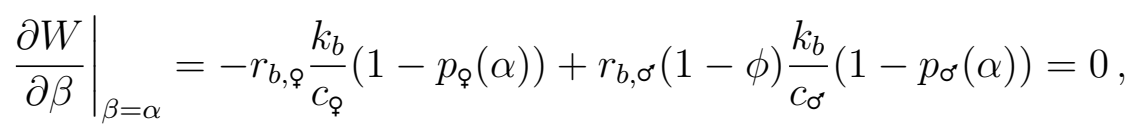

for both the NTI and TI models when we assume the reproductive functions are logistic. This condition was verified using the method discussed in appendix C, but using logistic reproductive functions. This condition is verified using the eigenvalues of the invasion matrix. 


\section{E.2 Evolutionarily Stable (ES)}

Using the method discussed in appendix D.2 we are able to determine whether an EE strategy, $\alpha^{*}$, is ES.

\section{E.2.1 NTI Model}

We use a computer algebra package to check the derivative condition in equation (D.2) assuming that all reproductive functions are logistic. To simplify the output we make use of the fact that all candidate ES strategies are EE. With this simplification, the resulting condition is

$$
\frac{4 k_{b}^{2}\left(1-s_{f}\right)^{2}\left(1-s_{b}\right)^{2}\left(1-p_{\sigma^{\star}}\right)^{2}\left(1-p_{\varphi}\right) p_{\varphi}^{2}}{c_{\sigma^{\prime}}^{2} c_{\varphi}\left(c_{\varphi} p_{\sigma^{\prime}}+c_{\sigma^{\circ}} p_{\varphi}-c_{\varphi}-c_{\sigma^{\prime}}\right)^{2}\left(p_{\varphi}+s_{b}-1\right)^{2}}\left\{\frac{1}{c_{\varphi}}\left(p_{\varphi}-\frac{1}{2}\right)+\frac{1}{c_{\sigma^{\circ}}}\left(p_{\sigma^{\circ}}-\frac{1}{2}\right)\right\} .
$$

Clearly, the term outside the curled braces in the above equation is always positive. Thus, $\alpha^{*}$ is ES in the NTI model when

$$
\frac{1}{c_{q}}\left(p_{\varphi}-\frac{1}{2}\right)+\frac{1}{c_{\sigma^{\prime}}}\left(p_{\sigma^{\prime}}-\frac{1}{2}\right)>0 .
$$

\section{E.2.2 TI Model}

Again, we use a computer algebra package to check the derivative condition in equation (D.3) assuming that all reproductive functions are logistic. We again can simplify the output since all ES strategies are EE. After this simplification, the resulting condition is

$$
\frac{4 k_{b}^{2} s_{b}^{2}\left(1-s_{f}\right)^{2}\left(1-s_{b}\right)^{2}\left(1-p_{\sigma^{\circ}}\right)^{2}\left(1-p_{q}\right) p_{q}^{2}}{c_{\sigma^{\prime}}^{2} c_{q}\left(c_{q} p_{\sigma^{\circ}}+c_{\sigma^{\circ}} p_{q}-c_{q}-c_{\sigma^{\prime}}\right)^{2}\left(p_{q}+s_{b}-1\right)^{2}}\left\{\frac{1}{c_{q}}\left(p_{q}-\frac{1}{2}\right)+\frac{1}{c_{\sigma^{\circ}}}\left(p_{\sigma^{\circ}}-\frac{1}{2}\right)\right\} .
$$


As with the NTI model the term outside the curled braces is always positive. Thus, $\alpha^{*}$ is ES in the TI model when

$$
\frac{1}{c_{\text {q }}}\left(p_{\text {क }}-\frac{1}{2}\right)+\frac{1}{c_{\sigma^{\circ}}}\left(p_{\text {○ั }}-\frac{1}{2}\right)>0 .
$$

\section{E.3 Convergence Stable (CS)}

We use the same method discussed in appendix D.3 to determine whether $\alpha^{*}$ is CS. Note that when we assume the reproductive functions are logistic the derivatives are

$$
\frac{d p_{\varphi}(X)}{d X}=-\frac{k_{b}}{c_{\varphi}} p_{\varphi}(X)\left(1-p_{\varphi}(X)\right) \quad \text { and } \quad \frac{d p_{\sigma^{\circ}}(X)}{d X}=\frac{k_{b}}{c_{\sigma^{\circ}}} p_{\sigma^{\prime}}(X)\left(1-p_{\sigma^{\prime}}(X)\right),
$$

where $X=\alpha, \beta$. We begin with the expression for fitness, $W$, found in the manuscript. The proof for CS is as follows:

$$
\begin{aligned}
& W=r_{b, q} \frac{p_{q}(\beta)}{p_{\uparrow}(\alpha)}+r_{b, \sigma^{*}}(1-\phi) \frac{p_{\sigma^{\circ}}(\beta)}{p_{\text {o }^{\prime}}(\alpha)} \\
& \left.\frac{\partial W}{\partial \beta}\right|_{\beta=\alpha}=-r_{b, q} \frac{k_{b}}{c_{\varphi}} \frac{p_{q}(\alpha)\left(1-p_{q}(\alpha)\right)}{p_{\varphi}(\alpha)}+r_{b, \sigma^{\circ}}(1-\phi) \frac{k_{b}}{c_{\sigma^{\prime}}} \frac{p_{\sigma^{\prime}}(\alpha)\left(1-p_{\sigma^{\circ}}(\alpha)\right)}{p_{\sigma^{\prime}}(\alpha)} \\
& \left.\frac{\partial W}{\partial \beta}\right|_{\beta=\alpha}=-r_{b, q} \frac{k_{b}}{c_{\varphi}}\left(1-p_{q}(\alpha)\right)+r_{b, \sigma^{\circ}}(1-\phi) \frac{k_{b}}{c_{\sigma^{\prime}}}\left(1-p_{\sigma^{*}}(\alpha)\right) \\
& \left.\frac{d}{d \alpha}\left[\left.\frac{\partial W}{\partial \beta}\right|_{\beta=\alpha}\right]_{\alpha=\alpha^{*}}=-r_{b, q} \frac{k_{b}^{2}}{c_{\varphi}^{2}} p_{\varphi}\left(\alpha^{*}\right)\left(1-p_{\varphi}\left(\alpha^{*}\right)\right)-r_{b, \sigma^{*}}(1-\phi) \frac{k_{b}^{2}}{c_{\sigma^{*}}^{2}} p_{\sigma^{*}}\left(\alpha^{*}\right)\left(1-p_{\sigma^{\circ}}\left(\alpha^{*}\right)\right)<0\right)
\end{aligned}
$$

Thus, $\alpha^{*}$ is always CS in both the NTI and TI models when we assume the reproductive functions are logistic.

\section{F Mutant Fitness for Helpful Behaviour}

Once again, the inclusion of self-fertilization causes us to consider both heterozygous, and homozygous mutant individuals in the invasion analysis. We assume that the breeder sex- 
allocation strategy is at its ES value, $\alpha^{*}$. By following a similar method as we did when studying the evolution of sex allocation, we are able to determine when helpful behaviour is selectively advantageous in a previously selfish population. In this scenario, the mutant allele causes a phenotypic deviation, $\varepsilon \geq 0$, in the offspring dispersal rate. This deviation cannot be negative since selfish individuals are not able to disperse more frequently than they already do. We define $d_{i}=1-\frac{i}{2} \varepsilon$, as the dispersal rate of an offspring from their natal patch. Once again, $i=0,1,2$ and is the number of copies of the mutant allele an individual has. We are interested in determining the condition for helpful behaviour to emerge in both the NTI and TI models. Unlike the prior sex allocation results, the conditions for invasion differ between the two models. Following the population dynamics set out in the main text, the mutant population can be described by nine compartments:

- the density of heterozygous floaters, $u_{1}$;

- the density of homozygous mutant floaters, $u_{2}$;

- the density of homozygous normal breeders with heterozygous helpers, $v_{01}$

- the density of solitary heterozygous breeders, $v_{1} \bullet$;

- the density of heterozygous breeders with heterozygous helpers, $v_{11}$;

- the density of heterozygous breeders with homozygous mutant helpers, $v_{12}$;

- the density of solitary homozygous mutant breeders, $v_{2} \bullet$;

- the density of homozygous mutant breeders with heterozygous helpers, $v_{21}$;

- the density of homozygous mutant breeders with homozygous mutant helpers, $v_{22}$; 


\section{F.1 Model I: No Territory Inheritance (NTI)}

We again follow the steps outline in Wild and Koykka (2014) for this invasion analysis. When there is no territory inheritance in the resident population the dynamics of the mutant population is described by,

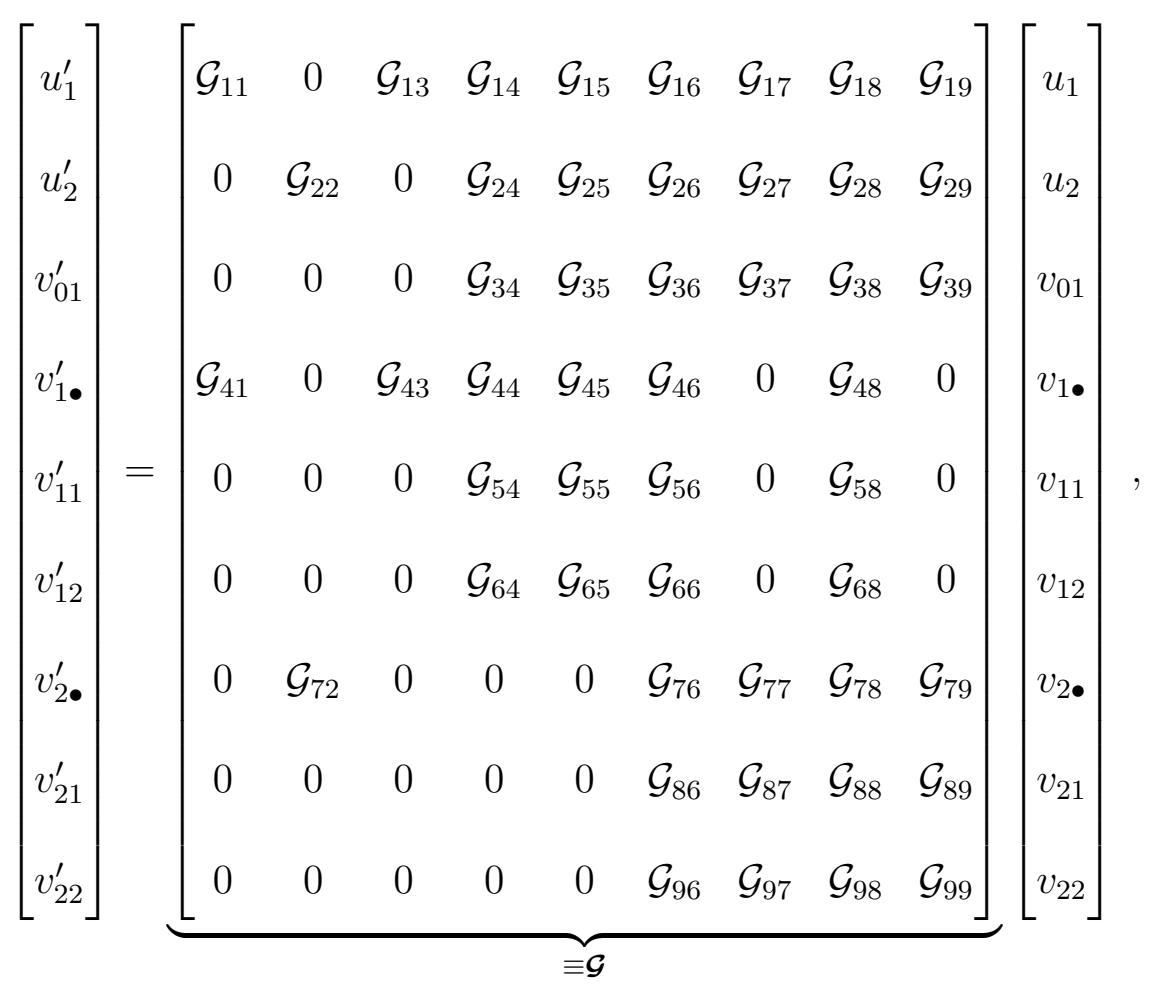

where prime denotes the next time step. The non-zero entries of $\mathcal{G}$ can be found in Tables 36. As a check we calculate the largest eigenvalue when there is no phenotypic change in the population (i.e., the mutant uses the same strategy as a normal individual, $\varepsilon=0$ ). We find $\lambda=1$ in this case, which is expected because an individual following the same strategy as in the resident population should neither be eliminated nor favoured by selection.

As before, we make an approximation to the long term geometric growth rate, $\lambda$, using a Taylor expansion and recall that mutant invasion occurs when $\lambda>1$. The approximation for the long term geometric growth rate for helpful behaviour is $\lambda \approx 1+\left.\frac{\varepsilon}{2} \frac{d \lambda}{d \varepsilon}\right|_{\varepsilon=0}$ plus 
terms of order $O\left(\varepsilon^{2}\right)$, which we can ignore because of the weak selection assumption. Since $\varepsilon \geq 0$, mutant invasion occurs when $\left.\frac{d \lambda}{d \varepsilon}\right|_{\varepsilon=0}$ is positive. To find this expression we implicitly differentiate the characteristic equation of $\mathcal{G}$ using a computer algebra package and set $\varepsilon=0$ and $\lambda=1$. For brevity, $p_{\varphi} \equiv p_{\varphi}\left(\alpha^{*}\right), q_{\varphi} \equiv q_{\varphi}\left(\alpha^{*}, \gamma\right), p_{\sigma^{*}} \equiv p_{\sigma^{*}}\left(\alpha^{*}\right)$, and $q_{\sigma^{*}} \equiv q_{\sigma^{*}}\left(\alpha^{*}, \gamma\right)$ below. We find,

$$
\begin{aligned}
& \left.\frac{d \lambda}{d \varepsilon}\right|_{\varepsilon=0}=\frac{1}{2-\phi} \frac{\left(1-s_{b}\right)\left(1-s_{f}\right) p_{\odot} s_{b}}{\left(2-s_{f}-s_{b}\right) p_{\varphi}-\left(1-s_{b}\right)^{2}} \\
& \left\{r_{h, q}\left(q_{\varphi}-p_{\varphi}\right)+r_{h, \sigma^{\circ}}(1-\phi)\left(\frac{q_{\sigma^{\circ}}}{p_{\sigma^{\circ}}}-1\right) p_{\varphi}+s_{h}\left(1-s_{b}\right)\left(\frac{p_{\odot}}{1-s_{b}}-1\right)-\left(1-s_{h}\right)\right\} .
\end{aligned}
$$

The term outside the curled brackets is always positive when $\mathcal{R}_{0}>1$ and the argument follows from the one presented in section C.1. The term in the curled brackets (note that it is the same as the one found in the main text) determines the sign of the selection gradient and when positive, mutant invasion occurs. 


\section{F.2 Model II: Territory Inheritance (TI)}

When there is territory inheritance in the normal population the dynamics of the mutant population is described by,

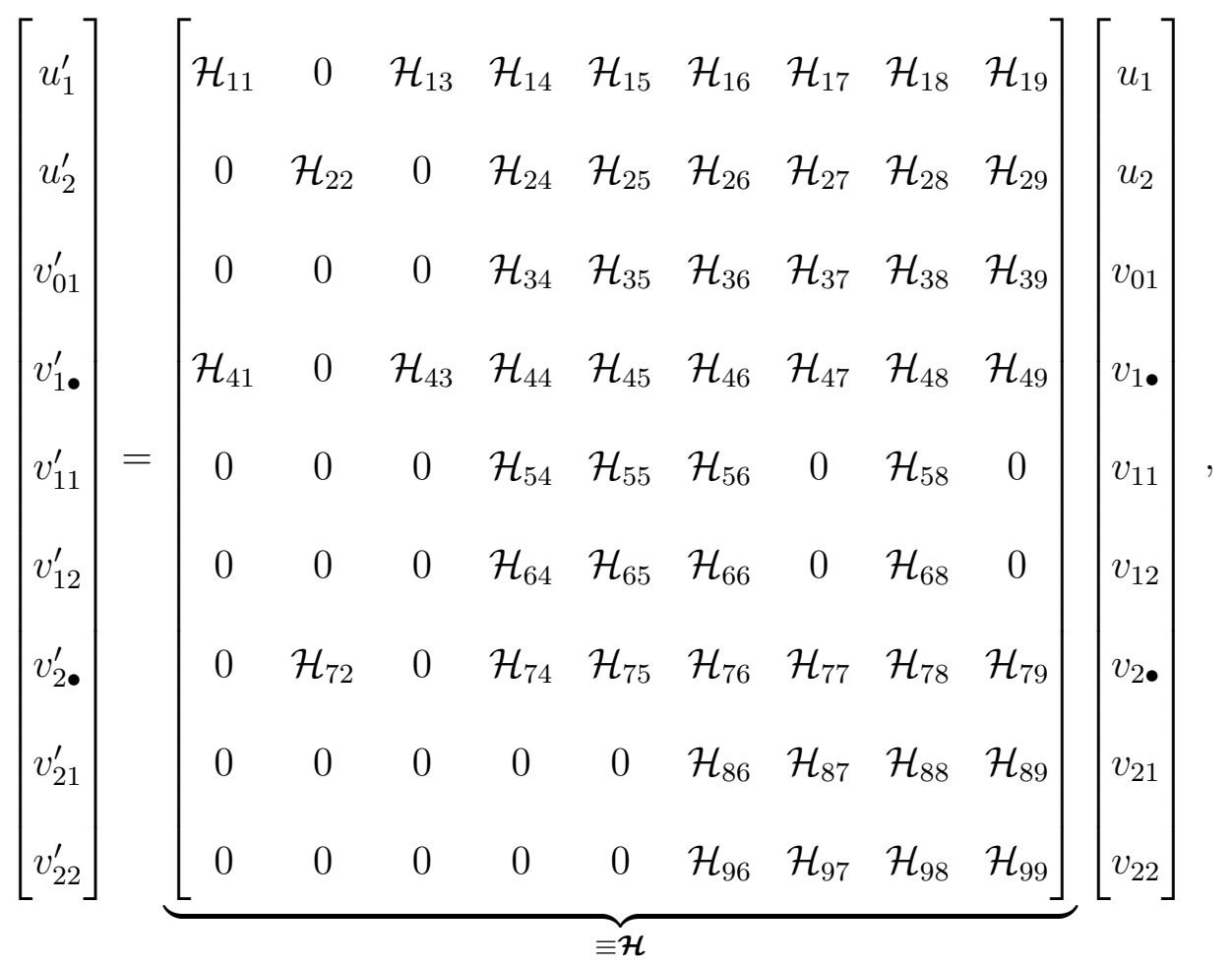

where prime denotes the next time ste. The non-zero entries of $\mathcal{H}$ can be found in Tables 7 . 11. To check if our model is biologically reasonable we calculate the largest eigenvalue when there is no phenotypic change in the population (i.e., the mutant uses the same strategy as a normal individual, $\varepsilon=0$ ). We find $\lambda=1$ in this case, which again is expected.

The approximation $\lambda \approx 1+\left.\frac{\varepsilon}{2} \frac{d \lambda}{d \varepsilon}\right|_{\varepsilon=0}$ is still used for the long-term geometric growth rate of the mutant population in this model. As before, the fact that $\varepsilon \geq 0$ causes mutant invasion to occur when $\left.\frac{d \lambda}{d \varepsilon}\right|_{\varepsilon=0}$ is positive. To find $\left.\frac{d \lambda}{d \varepsilon}\right|_{\varepsilon=0}$ we implicitly differentiate the characteristic equation of $\mathcal{H}$ using a computer algebra package and set $\varepsilon=0$ and $\lambda=1$. Once again for brevity, $p_{\varphi} \equiv p_{\varphi}\left(\alpha^{*}\right), q_{\varphi} \equiv q_{\phi}\left(\alpha^{*}, \gamma\right), p_{\sigma^{*}} \equiv p_{\sigma^{*}}\left(\alpha^{*}\right)$, and $q_{\sigma^{*}} \equiv q_{\sigma^{*}}\left(\alpha^{*}, \gamma\right)$ below. 
We find,

$$
\begin{aligned}
& \left.\frac{d \lambda}{d \varepsilon}\right|_{\varepsilon=0}=\frac{1}{2-\phi} \frac{\left(1-s_{b}\right)\left(1-s_{f}\right) s_{b}^{2} p_{q}}{\left(s_{b}^{2}-s_{b}\left(2+s_{f}\right)+2\right) p_{q}-\left(1-s_{b}\right)^{2}-\left(1-s_{b}\right) p_{q}^{2}} \\
& \left\{r_{h, q}\left(q_{q}-p_{q}\right)\left(1-s_{h}+s_{h} s_{b} v_{f}\right)+r_{h, \sigma^{\circ}}(1-\phi)\left(\frac{q_{\sigma^{\circ}}}{p_{\sigma^{\circ}}}-1\right) p_{\text {q }}\right. \\
& \left.+s_{h}\left(1-s_{b}\right)\left(v_{i}-v_{f}\right)-\left(1-s_{h}\right) v_{f}-s_{h}\left(1-s_{b}\right) r_{h, q}\left(p_{q} v_{i}-q_{q} v_{f}\right)\right\} .
\end{aligned}
$$

The term outside the curled brackets is always positive when $\mathcal{R}_{0}>1$ and the argument follows the one presented in section C.2. The term in the curled brackets (note that it is the same as the one found in the main text) determines the sign of the selection gradient and when positive, mutant invasion occurs.

\section{G Optimal Resource Allocation for a Helper}

Now we will go through our derivation for the optimal resource allocation, which we call $\gamma_{\max }$. We collect the terms of the inclusive fitness expression that the helper can directly influence (i.e., terms inside the curled brackets involving $q_{\phi}$ or $q_{\sigma^{\circ}}$ in equations $(\overline{F .2})$ and (F.4) $)$. The collection of terms is,

$$
M=v_{\varphi} r_{h, q} \frac{q_{\varphi}\left(\alpha^{*}, \gamma\right)}{p_{\varphi}\left(\alpha^{*}\right)}+v_{\diamond} r_{h, \sigma^{\circ}}(1-\phi) \frac{q_{\sigma^{*}}\left(\alpha^{*}, \gamma\right)}{p_{\sigma^{*}}\left(\alpha^{*}\right)},
$$

where $r_{h, q}=(1+\phi)^{2} / 4, r_{h, \sigma^{\circ}}=(1+\phi) / 4, v_{\sigma^{\circ}}=1$, and finally, in the NTI model $v_{q}=1$ and in the TI model $v_{q}=\left(1-s_{h}\right)+s_{h} v_{f}$. Recall $v_{f}=\frac{p_{q}}{1-s_{b}}$. To find the maximizing helper resource allocation, $\gamma_{\max }$, we take the first derivative with respect to this parameter, and solve for when the derivative condition is equal to zero. Note that $r_{h, q}=r_{b, q} r_{b, q}$ and $r_{h, \sigma^{*}}=r_{b, \sigma^{\circ}} r_{b, q}$ as discussed in Appendix B and recall $q_{q}\left(\alpha^{*}, \gamma\right)=1-\exp \left\{-\left(k_{b}\left(1-\alpha^{*}\right)+k_{h}(1-\gamma)\right) / c_{q}\right\}$ and 
$q_{\sigma^{*}}\left(\alpha^{*}, \gamma\right)=1-\exp \left\{-\left(k_{b} \alpha^{*}+k_{h} \gamma\right) / c_{\boldsymbol{o}^{*}}\right\}$. The derivative condition is,

$$
\begin{aligned}
\left.\frac{\partial M}{\partial \gamma}\right|_{\gamma=\gamma_{\max }}=r_{b, q}\left[-v_{\phi} r_{b, q}\left(\frac{k_{h}}{c_{\sigma^{*}}}\right)\right. & \frac{e^{-k_{b}\left(1-\alpha^{*}\right) / c_{q}}}{p_{\phi}\left(\alpha^{*}\right)} e^{-k_{h}\left(1-\gamma_{\max }\right) / c_{q}} \\
& \left.+v_{\sigma^{\circ}} r_{b, \sigma^{\circ}}(1-\phi)\left(\frac{k_{h}}{c_{\sigma^{\prime}}}\right) \frac{e^{-k_{b} \alpha^{*} / c_{\sigma^{*}}}}{p_{\sigma^{*}}\left(\alpha^{*}\right)} e^{-k_{h} \gamma_{\max } / c_{\sigma^{*}}}\right]=0 .
\end{aligned}
$$

Since the population is using strategy $\alpha^{*}$, the term in curled braces in equations C.2 and C.4 must be equal to zero. Consequently, we know,

$$
r_{b, q} \frac{e^{-k_{b}\left(1-\alpha^{*}\right) / c_{q}}}{p_{\wp}\left(\alpha^{*}\right)}=r_{b, \sigma^{*}}(1-\phi) \frac{e^{-k_{b} \alpha^{*} / c_{\sigma^{\circ}}}}{p_{\sigma^{*}}\left(\alpha^{*}\right)}=\zeta .
$$

The above expression is then substituted into equation G.1 and we now require,

$$
\zeta r_{b, \rho_{q}} k_{h}\left[-v_{q} e^{-k_{h}\left(1-\gamma_{\max }\right) / c_{q}}+v_{\sigma^{\circ}} e^{-k_{h} \gamma_{\max } / c_{\odot}}\right]=0 .
$$

Now we can explicitly solve for $\gamma_{\max }$ and it is,

$$
\gamma_{\max }=\min \left\{\frac{c_{\sigma^{\prime}}}{c_{\sigma^{\prime}}+c_{\sigma^{\prime}}}+\left(\frac{k_{h}}{c_{\sigma^{\prime}}}+\frac{k_{h}}{c_{\varphi}}\right)^{-1} \log \left(\frac{v_{\sigma^{\prime}}}{v_{\phi}}\right), 1\right\} .
$$

The minimum appears because mathematically $\gamma_{\max }$ cannot exceed 1 , but is not biologically reasonable. It is easy to verify that $\gamma_{\max }$ is indeed the helper sex allocation that provides the greatest inclusive fitness effect. This is proven by taking the partial derivative of equation G.2), (essentially the $2^{\text {nd }}$ derivative of $M$ ) with respect to $\gamma$. It follows trivially that,

$$
\begin{aligned}
\frac{\partial}{\partial \gamma} \zeta r_{b, q} k_{h}\left[-v_{q} e^{-k_{h}(1-\gamma) / c_{q}}+v_{\sigma^{\prime}} e^{-k_{h} \gamma / c_{\sigma^{\prime}}}\right] & \\
& =\zeta r_{b, q} k_{h}\left[-v_{\phi} \frac{k_{h}}{c_{q}} e^{-k_{h}(1-\gamma) / c_{q}}-v_{\sigma^{\prime}} \frac{k_{h}}{c_{\sigma^{*}}} e^{-k_{h} \gamma / c_{\sigma^{\prime}}}\right]<0 .
\end{aligned}
$$

Since the above condition is always negative we know $\gamma_{\max }$ maximizes equation G.1. 
Table 1: Model I - Expressions for non-zero entires in matrix $\mathcal{J}$ (equation (C.1)).

Matrix Entry Expression

Column 1

$\begin{array}{ll}\mathcal{J}_{11} & \left(1-\frac{1}{1+a \bar{B}}\right) s_{f} \\ \mathcal{J}_{31} & \frac{s_{f}}{1+a \bar{B}}\end{array}$

Column 2

$\begin{array}{ll}\mathcal{J}_{22} & \left(1-\frac{1}{1+a \bar{B}}\right) s_{f} \\ \mathcal{J}_{42} & \frac{s_{f}}{1+a \bar{B}}\end{array}$

Column 3

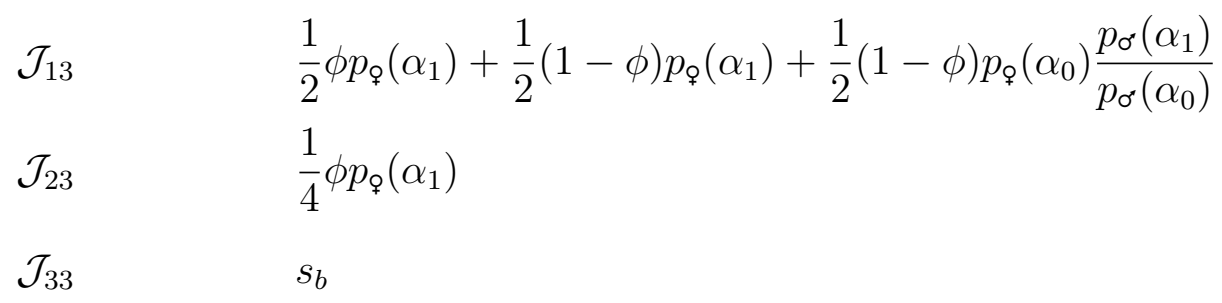

Column 4

$\begin{array}{ll}\mathcal{J}_{14} & (1-\phi) p_{\varphi}\left(\alpha_{2}\right)+(1-\phi) p_{\varphi}\left(\alpha_{0}\right) \frac{p_{\circlearrowleft^{*}}\left(\alpha_{2}\right)}{p_{\varsigma^{*}}\left(\alpha_{0}\right)} \\ \mathcal{J}_{24} & \phi p_{\varphi}\left(\alpha_{2}\right) \\ \mathcal{J}_{44} & s_{b}\end{array}$


Table 2: Model II - Expressions for non-zero entires in matrix $\mathcal{K}$ ( equation (C.3)).

Matrix Entry Expression

Column 1

$\mathcal{K}_{11} \quad\left(1-\frac{1}{1+a \bar{B}}\right) s_{f}$

$\mathcal{K}_{31} \quad \frac{s_{f}}{1+a \bar{B}}$

Column 2

$\begin{array}{ll}\mathcal{K}_{22} & \left(1-\frac{1}{1+a \bar{B}}\right) s_{f} \\ \mathcal{K}_{42} & \frac{s_{f}}{1+a \bar{B}}\end{array}$

Column 3

$\begin{array}{ll}\mathcal{K}_{13} & s_{b}\left(\frac{1}{2} \phi p_{\varphi}\left(\alpha_{1}\right)+\frac{1}{2}(1-\phi) p_{\varphi}\left(\alpha_{1}\right)+\frac{1}{2}(1-\phi) p_{\varphi}\left(\alpha_{0}\right) \frac{p_{\sigma^{\circ}}\left(\alpha_{1}\right)}{p_{\sigma^{\prime}}\left(\alpha_{0}\right)}\right) \\ \mathcal{K}_{23} & s_{b} \frac{1}{4} \phi p_{\varphi}\left(\alpha_{1}\right) \\ \mathcal{K}_{33} & s_{b}+\left(1-s_{b}\right)\left(\frac{1}{2} \phi p_{\varphi}\left(\alpha_{1}\right)+\frac{1}{2}(1-\phi) p_{\varphi}\left(\alpha_{1}\right)+\frac{1}{2}(1-\phi) p_{\varphi}\left(\alpha_{0}\right) \frac{p_{\sigma^{\prime}}\left(\alpha_{1}\right)}{p_{\sigma^{\prime}}\left(\alpha_{0}\right)}\right) \\ \mathcal{K}_{43} & \left(1-s_{b}\right) \frac{1}{4} \phi p_{\varphi}\left(\alpha_{1}\right)\end{array}$

Column 4
$\mathcal{K}_{14}$
$s_{b}\left((1-\phi) p_{\varphi}\left(\alpha_{2}\right)+(1-\phi) p_{\varphi}\left(\alpha_{0}\right) \frac{p_{\sigma^{\prime}}\left(\alpha_{2}\right)}{p_{\sigma^{\prime}}\left(\alpha_{0}\right)}\right)$
$\mathcal{K}_{24}$
$s_{b} \phi p_{\varphi}\left(\alpha_{2}\right)$
$\mathcal{K}_{34}$
$\left(1-s_{b}\right)\left((1-\phi) p_{\varphi}\left(\alpha_{2}\right)+(1-\phi) p_{\varphi}\left(\alpha_{0}\right) \frac{p_{\sigma^{\circ}}\left(\alpha_{2}\right)}{p_{\sigma^{\circ}}\left(\alpha_{0}\right)}\right)$
$\mathcal{K}_{44}$
$s_{b}+\left(1-s_{b}\right) \phi p_{\varphi}\left(\alpha_{2}\right)$ 
Table 3: Model I - Expressions for non-zero entires in matrix $\mathcal{G}$ (equation F.1)). Matrix Entry Expression

Column 1

$\begin{array}{ll}\mathcal{G}_{11} & \left(1-\frac{1}{1+a \bar{B}}\right) s_{f} \\ \mathcal{G}_{41} & \frac{s_{f}}{1+a \bar{B}}\end{array}$

Column 2

$\begin{array}{ll}\mathcal{G}_{22} & \left(1-\frac{1}{1+a \bar{B}}\right) s_{f} \\ \mathcal{G}_{72} & \frac{s_{f}}{1+a \bar{B}}\end{array}$

Column 3

$\begin{array}{ll}\mathcal{G}_{13} & s_{h} s_{b} \\ \mathcal{G}_{43} & s_{h}\left(1-s_{b}\right)\end{array}$

Column 4

\begin{tabular}{ll}
$\mathcal{G}_{14}$ & $\frac{1}{2} p_{\varphi}\left(1-\left(1-d_{1}\right) s_{b}\right)(2-\phi)$ \\
$\mathcal{G}_{24}$ & $\frac{1}{4} \phi p_{\varphi}\left(1-\left(1-d_{2}\right) s_{b}\right)$ \\
$\mathcal{G}_{34}$ & $\frac{1}{2}(1-\phi) p_{\varphi} s_{b}\left(1-d_{1}\right)$ \\
$\mathcal{G}_{44}$ & $s_{b}\left(1-\left(\frac{1}{4}\left(1-d_{2}\right) \phi+\frac{1}{2}\left(1-d_{1}\right)\right) p_{q}\right)$ \\
$\mathcal{G}_{54}$ & $\frac{1}{2} p_{\varphi} s_{b}\left(1-d_{1}\right)$ \\
$\mathcal{G}_{64}$ & $\frac{1}{4} \phi p_{q} s_{b}\left(1-d_{2}\right)$ \\
\hline
\end{tabular}


Table 4: Model I - Expressions for non-zero entires in matrix $\mathcal{G}$ (equation (F.1)).

\section{Matrix Entry Expression}

\section{Column 5}

$\begin{array}{ll}\mathcal{G}_{15} & \frac{1}{2}\left(1-\left(1-d_{1}\right)\left(\left(1-s_{h}\right) s_{b}+s_{h}\right)\right) q_{q}+s_{b} s_{h}+\frac{1}{2} \frac{q_{\sigma^{\circ}}}{p_{\sigma^{\circ}}}\left(1-\left(1-d_{1}\right) s_{b}\right)(1-\phi) p_{f} \\ \mathcal{G}_{25} & \frac{1}{4} \phi\left(1-\left(1-d_{2}\right)\left(s_{h}+(1-s) s_{b}\right)\right) q_{\odot} \\ \mathcal{G}_{35} & \frac{1}{2} \frac{q_{\sigma^{\circ}}}{p_{\sigma^{\circ}}}(1-\phi) p_{\odot} s_{b}\left(1-d_{1}\right) \\ \mathcal{G}_{45} & \frac{1}{4}\left(\left(1-s_{h}\right) s_{b}+s_{h}\right)\left(1-\left(\frac{1}{4}\left(1-d_{2}\right) \phi+\frac{1}{2}\left(1-d_{1}\right)\right) q_{\odot}\right) \\ \mathcal{G}_{55} & \frac{1}{2}\left(\left(1-s_{h}\right) s_{b}+s_{h}\right) q_{q}\left(1-d_{1}\right) \\ \mathcal{G}_{65} & \frac{1}{4}\left(\left(1-s_{h}\right) s_{b}+s_{h}\right) \phi q_{q}\left(1-d_{2}\right)\end{array}$

Column 6

\begin{tabular}{|c|c|}
\hline $\mathcal{G}_{16}$ & $\frac{1}{2} q_{q}\left(1-\left(1-d_{1}\right)\left(\left(1-s_{h}\right) s_{b}+s_{h}\right)\right)+\frac{1}{2} \frac{q_{\sigma^{\circ}}}{p_{\sigma^{\circ}}}\left(1-\left(1-d_{1}\right) s_{b}\right)(1-\phi) p_{q}$ \\
\hline $\mathcal{G}_{26}$ & $\frac{1}{4} \phi\left(1-\left(1-d_{2}\right)\left(\left(1-s_{h}\right) s_{b}+s_{h}\right)\right) q_{q}+s_{h} s_{b}$ \\
\hline $\mathcal{G}_{36}$ & $\frac{1}{2} \frac{q_{\sigma^{\circ}}}{p_{\sigma^{\circ}}}(1-\phi) p_{q} s_{b}\left(1-d_{1}\right)$ \\
\hline $\mathcal{G}_{46}$ & $\left(1-\left(\frac{1}{4}\left(1-d_{2}\right) \phi+\frac{1}{2}\left(1-d_{1}\right)\right) q_{q}\right) s_{b}$ \\
\hline $\mathcal{G}_{56}$ & $\frac{1}{2} q_{\varphi} s_{b}\left(1-d_{1}\right)$ \\
\hline $\mathcal{G}_{66}$ & $\frac{1}{4} \phi q_{q} s_{b}\left(1-d_{2}\right)$ \\
\hline $\mathcal{G}_{76}$ & $\left(1-\left(\frac{1}{4}\left(1-d_{2}\right) \phi+\frac{1}{2}\left(1-d_{1}\right)\right) q_{q}\right) s_{h}\left(1-s_{b}\right)$ \\
\hline $\mathcal{G}_{86}$ & $\frac{1}{2} q_{q} s_{h}\left(1-s_{b}\right)\left(1-d_{1}\right)$ \\
\hline $\mathcal{G}_{96}$ & $\frac{1}{4} \phi q_{q} s_{h}\left(1-s_{b}\right)\left(1-d_{2}\right)$ \\
\hline
\end{tabular}


Table 5: Model I - Expressions for non-zero entires in matrix $\mathcal{G}$ (equation (F.1)).

\begin{tabular}{|c|c|}
\hline Matrix Entry & Expression \\
\hline \multicolumn{2}{|l|}{ Column 7} \\
\hline $\mathcal{G}_{17}$ & $2(1-\phi) p_{q}\left(1-\left(1-d_{1}\right) s_{b}\right)$ \\
\hline $\mathcal{G}_{27}$ & $p_{q}\left(1-\left(1-d_{2}\right) s_{b}\right) \phi$ \\
\hline $\mathcal{G}_{37}$ & $(1-\phi) p_{\rho} s_{b}\left(1-d_{1}\right)$ \\
\hline $\mathcal{G}_{77}$ & $s_{b}\left(1-\left(1-(1-\phi) d_{1}-\phi d_{2}\right) p_{q}\right)$ \\
\hline $\mathcal{G}_{87}$ & $(1-\phi) p_{\rho} s_{b}\left(1-d_{1}\right)$ \\
\hline $\mathcal{G}_{97}$ & $\phi p_{q} s_{b}\left(1-d_{2}\right)$ \\
\hline \multicolumn{2}{|l|}{ Column 8} \\
\hline $\mathcal{G}_{18}$ & $\left(1-\left(1-d_{1}\right)\left(\left(1-s_{h}\right) s_{b}+s_{h}\right)\right) q_{q}(1-\phi)+s_{b} s_{h}+\frac{q_{\sigma^{\star}}}{p_{\sigma^{\circ}}}\left(1-\left(1-d_{1}\right) s_{b}\right)(1-\phi) p_{q}$ \\
\hline $\mathcal{G}_{28}$ & $\phi q_{\varphi}\left(1-\left(1-d_{2}\right)\left((1-s) s_{b}+s_{h}\right)\right)$ \\
\hline $\mathcal{G}_{38}$ & $\frac{q_{\sigma^{*}}}{p_{\sigma^{*}}}(1-\phi) p_{\rho} s_{b}\left(1-d_{1}\right)$ \\
\hline $\mathcal{G}_{48}$ & $\left(1-\left(1-(1-\phi) d_{1}-\phi d_{2}\right) q_{q}\right) s_{h}\left(1-s_{b}\right)$ \\
\hline $\mathcal{G}_{58}$ & $(1-\phi) q_{q} s\left(1-s_{b}\right)\left(1-d_{1}\right)$ \\
\hline $\mathcal{G}_{68}$ & $\phi q_{q} s_{h}\left(1-s_{b}\right)\left(1-d_{2}\right)$ \\
\hline $\mathcal{G}_{78}$ & $\left(1-\left(1-(1-\phi) d_{1}-\phi d_{2}\right) q_{\varphi}\right) s_{b}$ \\
\hline $\mathcal{G}_{88}$ & $(1-\phi) q_{q} s_{b}\left(1-d_{1}\right)$ \\
\hline $\mathcal{G}_{98}$ & $\phi q_{\odot} s_{b}\left(1-d_{2}\right)$ \\
\hline
\end{tabular}


Table 6: Model I - Expressions for non-zero entires in matrix $\mathcal{G}$ (equation (F.1)).

\section{Matrix Entry Expression}

Column 9

\begin{tabular}{ll}
$\mathcal{G}_{19}$ & $\left(1-\left(1-d_{1}\right)\left(\left(1-s_{h}\right) s_{b}+s_{h}\right)\right) q_{\varphi}(1-\phi)+\frac{q_{\sigma^{\circ}}}{p_{\sigma^{\circ}}}\left(1-\left(1-d_{1}\right) s_{b}\right)(1-\phi) p_{f}$ \\
$\mathcal{G}_{29}$ & $\phi q_{\varphi}\left(1-\left(1-d_{2}\right)\left(\left(1-s_{h}\right) s_{b}+s_{h}\right)\right)+s_{h} s_{b}$ \\
$\mathcal{G}_{39}$ & $\frac{q_{\sigma^{\circ}}}{p_{\sigma^{\circ}}}(1-\phi) p_{\varphi} s_{b}\left(1-d_{1}\right)$ \\
$\mathcal{G}_{79}$ & $\left(1-\left(1-(1-\phi) d_{1}-\phi d_{2}\right) q_{\varphi}\right)\left(\left(1-s_{h}\right) s_{b}+s_{h}\right)$ \\
$\mathcal{G}_{89}$ & $(1-\phi)\left(1-d_{1}\right) q_{q}\left(\left(1-s_{h}\right) s_{b}+s_{h}\right)$ \\
$\mathcal{G}_{99}$ & $\phi\left(1-d_{2}\right) q_{\varphi}\left(\left(1-s_{h}\right) s_{b}+s_{h}\right)$ \\
\hline
\end{tabular}


Table 7: Model II - Expressions for non-zero entires in matrix $\mathcal{H}$ (equation (F.3)).

Matrix Entry Expression

Column 1

$\mathcal{H}_{11} \quad\left(1-\frac{1}{1+a \bar{B}}\right) s_{f}$

$\mathcal{H}_{41} \quad \frac{s_{f}}{1+a \bar{B}}$

Column 2

$\begin{array}{ll}\mathcal{H}_{22} & \left(1-\frac{1}{1+a \bar{B}}\right) s_{f} \\ \mathcal{H}_{72} & \frac{s_{f}}{1+a \bar{B}}\end{array}$

Column 3

$\mathcal{H}_{13} \quad s_{h} s_{b}$

$\mathcal{H}_{43} \quad s_{h}\left(1-s_{b}\right)$

Column 4

$\begin{array}{ll}\mathcal{H}_{14} & \frac{1}{2} p_{\varphi} s_{b} d_{1}(2-\phi) \\ \mathcal{H}_{24} & \frac{1}{4} \phi p_{\varphi} s_{b} d_{2} \\ \mathcal{H}_{34} & \frac{1}{2}(1-\phi) p_{\varphi} s_{b}\left(1-d_{1}\right) \\ \mathcal{H}_{44} & \frac{1}{2} p_{\varphi} s_{b} d_{1}+\frac{1}{4} \phi p_{\varphi} s_{b} d_{2}+\left(1-p_{\varphi}\right) s_{b}+\frac{1}{2} \phi p_{\varphi}\left(1-s_{b}\right)+(1-\phi) p_{\varphi}\left(1-s_{b}\right)+ \\ & \frac{1}{2}(1-\phi) p_{\varphi} s_{b}+\frac{1}{4} \phi p_{\varphi} s_{b} \\ & \frac{1}{2} p_{\varphi} s_{b}\left(1-d_{1}\right) \\ \mathcal{H}_{54} & \frac{1}{4} \phi p_{\varphi} s_{b}\left(1-d_{2}\right) \\ \mathcal{H}_{64} & \frac{1}{4} \phi p_{\varphi}\left(1-s_{b}\right) \\ \mathcal{H}_{74} & \end{array}$


Table 8: Model II - Expressions for non-zero entires in matrix $\mathcal{H}$ (equation $(\overline{\text { F.3p) }}$ ).

Matrix Entry Expression

Column 5

$\begin{array}{ll}\mathcal{H}_{15} & \frac{1}{2} q_{\varphi}\left((1-s) s_{b}+s_{h}\right) d_{1}+s s_{b}+\frac{1}{2} \frac{q_{\sigma^{\circ}}}{p_{\sigma^{\circ}}}(1-\phi) p_{q} s_{b} d_{1} \\ \mathcal{H}_{25} & \frac{1}{4} \phi d_{2} q_{\varphi}\left(\left(1-s_{h}\right) s_{b}+s_{h}\right) \\ \mathcal{H}_{35} & \frac{1}{2} \frac{q_{\sigma^{\circ}}}{p_{\sigma^{\circ}}}(1-\phi) p_{\varphi} s_{b}\left(1-d_{1}\right) \\ \mathcal{H}_{45} & \frac{1}{2} \frac{q_{\sigma^{\circ}}}{p_{\sigma^{\circ}}}(1-\phi) p_{\varphi}\left(1-s_{b}\right)+q_{f}\left(\left(1-s_{h}\right) s_{b}+s_{h}\right)\left(\frac{1}{2} d_{1}+\frac{1}{4} \phi d_{2}\right)+\frac{1}{2} q_{q}+ \\ & \left(s_{h}+\left(1-s_{h}\right) s_{b}\right)\left(1-\frac{1}{4}(\phi+4) q_{\varphi}\right) \\ \mathcal{H}_{55} & \frac{1}{2}\left(1-d_{1}\right) q_{\varphi}\left(\left(1-s_{h}\right) s_{b}+s_{h}\right) \\ \mathcal{H}_{65} & \frac{1}{4} \phi\left(1-d_{2}\right) q_{\varphi}\left(\left(1-s_{h}\right) s_{b}+s_{h}\right) \\ \mathcal{H}_{75} & \frac{1}{4} \phi q_{\varphi}\left(1-s_{h}\right)\left(1-s_{b}\right)\end{array}$


Table 9: Model II - Expressions for non-zero entires in matrix $\mathcal{H}$ (equation $(\overline{F .3 p})$.

\section{Matrix Entry Expression}

Column 6

\begin{tabular}{|c|c|}
\hline $\mathcal{H}_{16}$ & $\frac{1}{2} \frac{q_{\sigma^{\star}}}{p_{\sigma^{\prime}}}(1-\phi) p_{\varphi} s_{b} d_{1}+\frac{1}{2} q_{\varphi}\left(\left(1-s_{h}\right) s_{b}+s_{h}\right) d_{1}$ \\
\hline $\mathcal{H}_{26}$ & $\frac{1}{4} \phi d_{2} q_{q}\left(\left(1-s_{h}\right) s_{b}+s_{h}\right)+s s_{b}$ \\
\hline $\mathcal{H}_{36}$ & $\frac{1}{2} \frac{q_{\sigma^{\star}}}{p_{\sigma^{\prime}}}(1-\phi) p_{\varphi} s_{b}\left(1-d_{1}\right)$ \\
\hline $\mathcal{H}_{46}$ & $\begin{array}{l}\frac{1}{2} \frac{q_{\sigma^{*}}}{p_{\sigma^{\prime}}}(1-\phi) p_{q}\left(1-s_{b}\right)+\phi q_{f} s_{b}\left(\frac{1}{2} d_{1}+\frac{1}{4} d_{2}\right)+\frac{1}{2}\left(1-s_{h}\right)\left(1-s_{b}\right) q_{q}+ \\
\left(1-\frac{1}{4} q_{q}(2+\phi)\right) s_{b}\end{array}$ \\
\hline $\mathcal{H}_{56}$ & $\frac{1}{2} q_{\phi} s_{b}\left(1-d_{1}\right)$ \\
\hline $\mathcal{H}_{66}$ & $\frac{1}{4} \phi q_{q} s_{b}\left(1-d_{2}\right)$ \\
\hline $\mathcal{H}_{76}$ & $\begin{array}{l}\frac{1}{2}\left(1-s_{b}\right)\left(\frac{1}{2} \phi q_{q}+s_{h}\left(1-\phi q_{q}\right)+s_{h}\left(1-q_{q}\right)\right)+\frac{1}{2} s_{h} q_{f} d_{1}\left(1-s_{b}\right)+ \\
\frac{1}{4} \phi q_{f} s_{h}\left(1-s_{b}\right) d_{2}\end{array}$ \\
\hline $\mathcal{H}_{86}$ & $\frac{1}{2} q_{\odot} s_{h}\left(1-s_{b}\right)\left(1-d_{1}\right)$ \\
\hline $\mathcal{H}_{96}$ & $\frac{1}{2} \phi q_{\phi} s_{h}\left(1-s_{b}\right)\left(1-d_{2}\right)$ \\
\hline
\end{tabular}


Table 10: Model II - Expressions for non-zero entires in matrix $\mathcal{H}$ (equation $(\overline{F .3 p})$.

Matrix Entry Expression

Column 7

$\begin{array}{ll}\mathcal{H}_{17} & 2(1-\phi) p_{q} s_{b} d_{1} \\ \mathcal{H}_{27} & \phi p_{\varphi} s_{b} d_{2} \\ \mathcal{H}_{37} & (1-\phi) p_{q} s_{b}\left(1-d_{1}\right) \\ \mathcal{H}_{47} & 2(1-\phi) p_{q}\left(1-s_{b}\right) \\ \mathcal{H}_{77} & (1-\phi) s_{b}\left(p_{q} d_{1}+1-p_{q}\right)+\phi p_{\varphi}\left(1-s_{b}+s_{b} d_{2}\right)+\phi\left(1-p_{q}\right) s_{b} \\ \mathcal{H}_{87} & (1-\phi) p_{\varphi} s_{b}\left(1-d_{1}\right) \\ \mathcal{H}_{97} & \phi p_{\varphi} s_{b}\left(1-d_{2}\right)\end{array}$

Column 8

\begin{tabular}{ll}
$\mathcal{H}_{18}$ & $\frac{q_{\sigma^{\circ}}}{p_{\sigma^{\circ}}}(1-\phi) p_{\varphi} s_{b} d_{1}+\left(\left(1-s_{h}\right) s_{b}+s_{h}\right)(1-\phi) q_{q} d_{1}+s s_{b}$ \\
$\mathcal{H}_{28}$ & $\phi d_{2} q_{\varphi}\left(\left(1-s_{h}\right) s_{b}+s_{h}\right)$ \\
$\mathcal{H}_{38}$ & $\frac{q_{\sigma^{\circ}}}{p_{\sigma^{\circ}}}(1-\phi) p_{\varphi} s_{b}\left(1-d_{1}\right)$ \\
$\mathcal{H}_{48}$ & $\frac{q_{\sigma^{\circ}}}{p_{\sigma^{\circ}}}(1-\phi) p_{\varphi}\left(1-s_{b}\right)+(1-\phi) q_{q} s_{h}\left(1-s_{b}\right) d_{1}+\phi q_{q} s_{h}\left(1-s_{b}\right) d_{2}+$ \\
& $\left(1-s_{b}\right)\left((1-\phi) q_{\varphi}\left(1-s_{h}\right)+s_{h}\left(1-q_{q}\right)\right)$ \\
$\mathcal{H}_{58}$ & $(1-\phi) q_{\odot} s_{h}\left(1-s_{b}\right)\left(1-d_{1}\right)$ \\
$\mathcal{H}_{68}$ & $\phi q_{q} s_{h}\left(1-s_{b}\right)\left(1-d_{2}\right)$ \\
$\mathcal{H}_{78}$ & $q_{f} s_{b} d_{1}(1-\phi)+\phi q_{f} s_{b} d_{2}+\phi q_{\varphi}\left(1-s_{h}\right)\left(1-s_{b}\right)+\left(1-q_{f}\right) s_{b}$ \\
$\mathcal{H}_{88}$ & $(1-\phi) q_{q} s_{b}\left(1-d_{1}\right)$ \\
$\mathcal{H}_{98}$ & $\phi q_{q} s_{b}\left(1-d_{2}\right)$ \\
\hline
\end{tabular}


Table 11: Model II - Expressions for non-zero entires in matrix $\mathcal{H}$ (equation (F.3)).

\section{Matrix Entry Expression}

Column 9

\begin{tabular}{ll}
$\mathcal{H}_{19}$ & $\frac{q_{\sigma^{\circ}}}{p_{\sigma^{\circ}}}(1-\phi) p_{\varphi} s_{b} d_{1}+\left(\left(1-s_{h}\right) s_{b}+s_{h}\right)(1-\phi) q_{q} d_{1}$ \\
$\mathcal{H}_{29}$ & $\phi d_{2} q_{q}\left(\left(1-s_{h}\right) s_{b}+s_{h}\right)+s_{h} s_{b}$ \\
$\mathcal{H}_{39}$ & $\frac{q_{\sigma^{\circ}}}{p_{\sigma^{\circ}}}(1-\phi) p_{\varphi} s_{b}\left(1-d_{1}\right)$ \\
$\mathcal{H}_{49}$ & $(1-\phi) q_{\varphi}\left(1-s_{h}\right)\left(1-s_{b}\right)+\frac{q_{\sigma^{*}}}{p_{\sigma^{\circ}}}(1-\phi) p_{\varphi}\left(1-s_{b}\right)$ \\
$\mathcal{H}_{79}$ & $d_{1}(1-\phi) q_{q}\left(\left(1-s_{h}\right) s_{b}+s_{h}\right) \quad\left(\left(1-s_{h}\right) s_{b}+s_{h}\right) q_{q} \phi d_{2}$ \\
& $\phi q_{q}\left(1-s_{h}-\left(1-s_{h}\right) s_{b}\right)+\left(s_{h}+\left(1-s_{h}\right) s_{b}\right)\left(1-q_{q}\right)$ \\
$\mathcal{H}_{89}$ & $\left(1-d_{1}\right)(1-\phi) q_{\varphi}\left(\left(1-s_{h}\right) s_{b}+s_{h}\right)$ \\
$\mathcal{H}_{99}$ & $\phi\left(1-d_{2}\right) q_{q}\left(\left(1-s_{h}\right) s_{b}+s_{h}\right)$ \\
\hline
\end{tabular}

